\title{
Copper and Uranium Deposits of the Coyote District, Mora County New Mexico
}

GEOLOGICAL SURVEY BULLETIN 1030 -L

This report concerns work done on behalf of the U.S. Atomic Energy Commission and is published with the permission of the Commission 


\section{Copper and Uranium}

\section{Deposits of the Coyote}

District, Mora County

\section{New Mexico}

By C. M. TSCHANZ, D. C. LAUB, and G. W. FULLER

CONTRIBUTIONS TO THE GEOLOGY OF URANIUM

GE OLOGICAL SURVEY B ULLETIN $1030-\mathrm{L}$

This report concerns work done on behalf of the U.S. Atomic Energy Commission and is published with the permission of the Commission

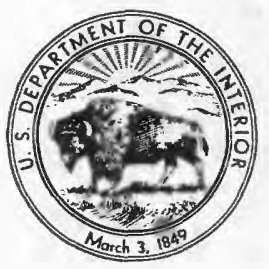


UNITED STATES DEPARTMENT OF THE INTERIOR

FRED A. SEATON, Secretary

\author{
GEOLOGICAL SURVEY
}

Thomas B. Nolan, Director 


\section{CONTENTS}

Page

Abstract

Introduction ...

Location and accessibility . _

Topography . . . . . . 344

Previous work ..... 345

Purpose and methods. . .

Acknowledgments . . . .

Geology ...

Geologic history

Stratigraphy _...

Precambrian rocks

Magdalena group

Description........ 349

Fossils . .

Sangre de Cristo formation

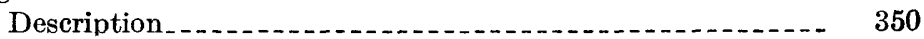

Fossils _...

Conditions of deposition........ 354

Yeso formation

San Andres formation (Glorieta sandstone member)

Bernal formation . . .

Santa Rosa sandstone...

Quaternary(?) basalt._.

Structure

Mineral deposits...... 356

Copper deposits

Description . .

Mineralogy

Uranium deposits..................... 370

Uranium deposits in sandstone..... 370

Area $B$, Blas Medina property ... 370

Area $F$, Arturo Le Deux property . . . 378

Uraniferous siltstone and shale.

Mineralogy of uranium deposits..................... 379

Chemical characteristics of uranium-bearing sandstone..... $\quad 380$

Geochemical prospecting . . 38

Origin ........ 383

Copper deposits.... 383

Uranium deposits_... . .

Uraniferous siltstone $\ldots \ldots \ldots \ldots$

Uranium-vanadium deposits in sandstone........ 388

Summary _... _.. _. .

Comparison with other copper-uranium deposits........ 391

Giuides to ore deposits.......... 392

Conclusions

Literature cited

Index . . . 


\section{ILLUSTRATIONS}

[Plates 24-32 in pocket]

Plate 24. Generalized geologic map.

25. Geologic map of area $A$.

26. Geologic map of area $B$.

27. Geologic map of area $C$.

28. Geologic map of area $E$.

29. Geologic map of area $F$.

30. Geologic map of area $G$.

31. Geologic map of area $H$.

32. Geologic map of area $I$.

33. Photomicrographs of copper ores. . . . . . . . . . . . Facing

34. Photomicrographs of uraniferous sandstone........ Facing

Figdre 67. Index map of Mora County, N. Mex

68. Columnar section of sedimentary rocks .................. 348

69. Columnar section of Sangre de Cristo formation........... 351

70. Columnar section of main ore-bearing interval ........... 352

71. Section showing relationship of copper to rock types, area $H_{-}-367$

72. Section showing concentration of copper and uranium in carbonaceous shale, area $H$

73. Section along ore sandstone, Blas Medina property _...... 370

\section{TABLES}

TABLE 1. Descriplion of the Sangre de Cristo formation...............

2. Marine fossils of the Sangre de Cristo formation

3. Analytical data for samples from area $A$ (pl. 25)

4. Analytical data for samples from area $B$ (pl. 26)

5. Analytical data for samples from area $C$ (pl. 27)

6. Analytical data for samples from area $E$ (pl. 28) . . . . . . . 361

7. Analytical data for samples from area $F$ (pl. 29) . . . . . . 362

8. Analytical data for samples from areas $G$ and $H$ (pls. 30 and 31)

9. Analytical data for samples from area $I$ (pl. 32)

10. Analytical data for samples outside areas of large-scale maps.-

11. Spectrographic, chemical, and radioactivity analyses of selected copper and uranium samples.

12. Chemical characteristics of uranium-bearing sandstone 


\title{
CONTRIBUTIONS TO THE GEOLOGY OF URANIUM
}

\section{COPPER AND URANIUM DEPOSITS OF THE COYOTE DISTRICT, MORA COUNTY, NEW MEXICO}

\author{
By C. M. Tschanz, D. C. LadB, and G. W. Foluer
}

\section{ABSTRACT}

The copper and uranium-vanadium deposits of the Coyote district, Mora County, N. Mex., are confined to the lower 2,000 feet of the Sangre de Cristo formation of Pennsylvanian and Permian(?) age. A narrow belt of deposits in steeply dipping or overturned rocks extends for 7 miles along Coyote Creek south of Guadalupita.

Earlier studies showed that the copper deposits contained uranium, but the reserves and the uranium content of the copper-bearing shale are too small to justify any attempt to recover uranium. Several small, commercial-grade uranium deposits have been discovered in sandstone, however.

Small isolated lenses of copper-bearing carbonaceous shale, siltstone, limestone or sandstone, interbedded with predominantly red rocks, are present at 12 or more stratigraphic levels. The better deposits, in carbonaceous shale, average about 2 percent copper. The copper content of the other rocks is usually lower, but small concentrations may contain 6 percent copper. The principal copper minerals are chalcocite and malachite. Chalcocite replaces wood and forms nodules that contain small, variable amounts of pyrite, bornite, covellite, and, rarely, uraninite.

The uranium deposits occur as small, closely spaced pockets that are commonly localized by sedimentary structures within one or more fluviatile arkosic sandstone beds near the middle of the formation, particularly where carbonized wood and clay and rock fragments are abundant. The uraniferous sandstone is commonly stained pink by hematite that was probably introduced with the uranium. The color increases in intensity with the radioactivity.

The outcrops of the uranium deposits are typically inconspicuous, but close inspection reveals malachite, chalcopyrite, black vanadium minerals of micaceous habit, metatyuyamunite, and microscopic grains of an unidentified black uraniferous substance. The proportion of copper, uranium, and vanadium is variable and any of these metals may be dominant in the ore.

The metals probably were derived from Precambrian granitic rocks. Copper and minor amounts of uranium were deposited in local stagnant basins by reaction with hydrogen sulfide and decaying organic material. The uraniferous shale and the copper deposits are probably syngenetic, or nearly so, but the uranium deposits in sandstone are epigenetic and probably were deposited from ground waters with a possible hydrothermal admixture. The uranium and vanadium may have been reconcentrated from earlier, low-grade, syngenetic deposits.

The average copper ore body assays about 1.5 percent copper and contains less than 1,000 tons. The small size of known, commercial-grade uranium pockets 
and the small total uranium reserves will permit small-scale mining only under favorable conditions. Further exploration probably would result in an increase of uranium reserves, but no large deposits are expected.

\section{INTRODUCTION}

\section{LOCATION AND ACCESSIBILITY}

The Coyote district is in Mora County in the north-central part of New Mexico (fig. 67). The district can be reached from Las Vegas,

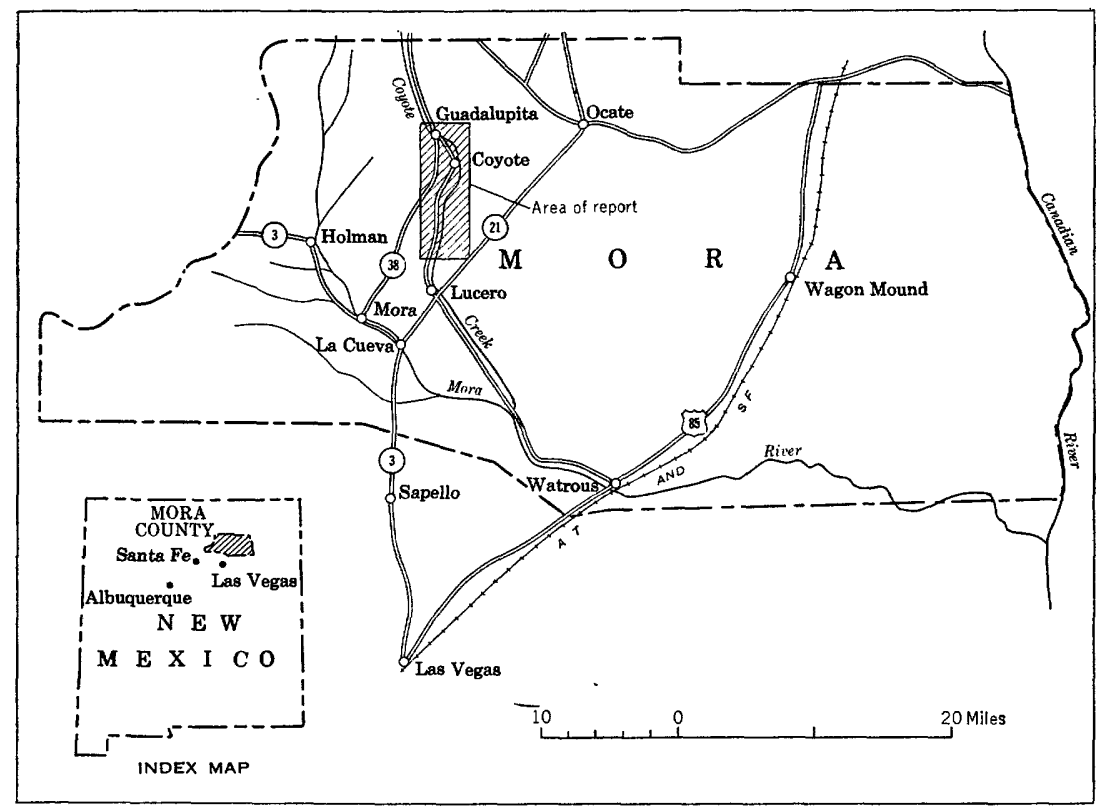

Figurm 67,--Index map of Mora County, N. Mex.

N. Mex., by traveling 28 miles north on State Route 3 to Mora, 8 miles north on State Route 38, and 2 miles east on an unimproved road. The district can also be reached by turning off State Route 3 at La Cueva and following State Route 21 for 6 miles, 2 miles west to Lucero on an unimproved road and 3 miles north along Coyote Creek. The roads in the district are ungraded and in poor condition. The nearest railroad shipping points are Watrous and Las Vegas. The nearest uranium mill and buying station is at Ship Rock, N. Mex. Sufficient water is probably available in Coyote Creek to operate a small mill.

\section{TOPOGRAPHY}

The narrow north-south valley of Coyote Creek approximates the boundary between the gently dipping Permian and Mesozoic rocks of the High Plains to the east and the Pennsylvanian and Precambrian 
rocks of the Southern Rocky Mountains to the west. The district is on the east flank of a north-south hogback between Coyote Creek on the east and Turquillo Valley on the west.

\section{PREVIOUS WORK}

The copper deposits of the district have been known for 50 years. Brief descriptions of the district have been published by Lindgren, Graton, and Gordon (1910, p. 109) ; Lasky and Wooton (1933, p. 84); Harley (1940, p. 42-43). In 1944 Read, Sample, and Shelton (written communication, 1954) examined the deposits and described them. Bachman and Read (written communication, 1952) discovered radioactive material in the district in 1951. Gott and Erickson (1952) visited the district and showed that the radioactivity was caused by uranium. As a result, the Geological Survey began an intensive study of the district for the Atomic Energy Commission, first by Zeller and Baltz (1954) and later by the writers of this report.

The district is shown on a geologic map of the Ocate area by Bachman (1953). A generalized topographic and geologic map of most of the district on a scale of $1: 12,000$ is included in a report by Zeller and Baltz (1954, fig. 4).

\section{PURPOSE AND METHODS}

Preliminary work in the Coyote district in 1952 showed that widespread copper-bearing carbonaceous shale contained small amounts of uranium, indicating the possibility of very large reserves of low-grade copper ores from which uranium might be recovered as a byproduct. The present study was begun primarily to explore this possibility. A secondary objective was the gathering of data on the origin of the ore deposits and the relationship between the copper and uranium deposits. As work progressed, the uranium content of the copper-bearing shale was found to be negligible and the plan was revised to include evaluation of newly discovered uranium-vanadium deposits in sandstone.

The most promising areas were mapped on a scale of 1 inch to 200 feet. Two small areas were mapped on a scale of 1 inch to 50 feet. The total area mapped was about 1.5 square miles. The most promising deposits were explored by about 200 shallow hand-dug trenches and 25 shallow jeep-mounted-auger holes. The trenches were sampled and mapped on a scale of 1 inch to 5 feet. A total of 339 soil samples and 457 botanical samples were collected. Wherever possible, soil and botanical samples were collected at the same site to permit comparison of the results of the two prospecting methods.

Three hundred fifty-one samples were collected from the copper and uranium deposits of which 210 samples were submitted for chemical 
analyses, 22 for semiquantitative spectrographic analyses, and 5 for mineral identification by X-ray diffraction techniques. About 70 polished sections, 6 thin sections, and several heavy-mineral concentrates were studied. Four autoradiographs were made and studied. Several samples of uranium ore have been submitted for age determination but the results are not yet available. The field work was accomplished during 5 months in the summer and fall of 1953 and 2 weeks in the following spring.

\section{ACKNOWLEDGMENTS}

All analytical work was done in the laboratories of the U. S. Geological Survey. The spectrographic analyses were made by G. W. Boyes and P. J. Dunton, the X-ray analyses by W. F. Outerbridge, the equivalent uranium determinations by S. P. Furman and E. J. Fennelly, and the selenium analyses were made by J. L. Siverly. Chemical analyses for copper, uranium, and vanadium were made by $\mathrm{E}$. C. Mallory, J. W. Patton, W. D. Goss, J. F. Wahlberg, J. P. Schuch, Wayne Mountjoy, D. L. Stockwell, E. J. Fennelly, H. E. Bivens, J. E. Wilson, D. L. Skinner, and R. F. Dufour. Rapid colorimetric analyses for trace amounts of copper and zinc were made by $\mathrm{H}$. $\mathrm{E}$. Crowe and J. H. McCarthy.

The plant and soil samples were collected by W. R. Martin. The writers are indebted to H. D. Zeller and E. H. Baltz, Jr., who kindly loaned their maps, notes, and specimens, and to J. G. McGrath for operation of the jeep-mounted auger.

This work is part of the program of exploration for radioactive raw materials undertaken by the Geological Survey on behalf of the U.S. Atomic Energy Commission.

\section{GEOLOGY}

\section{GEOLOGIC HISTORY}

During Pennsylvanian and early Permian time, the Ocate-Guadalupita area was part of a geosyncline, the Rowe-Mora trough, which was flanked by rising geanticlines of Precambrian rocks that were part of the Colorado Mountains or ancestral Rocky Mountains (Read and Wood, 1947, p. 266). The axis of the geosyncline, which trends northwest, was about 12 miles west of the district. The rising positive areas furnished large volumes of sediments to the basin, which was occupied by a sea during the deposition of most of the Magdalena group. Marine sedimentation recurred intermittently during the deposition of the lower part of the Sangre de Cristo formation, but most of the sediments were deposited under subaerial conditions.

The sea invaded the region repeatedly after the deposition of the Sangre de Cristo formation. Bachman (1953) believes that the 
Glorieta sandstone member of the San Andres formation of late Permian age is probably a beach sand deposited as the sea transgressed northward. Triassic and Jurassic sediments are partly shallow marine and partly continental in origin. A major marine transgression lasted during most of Cretaceous time.

In early Tertiary time, regional compression deformed the rocks in the district and buckled the site of the present Sangre de Cristo Mountain system into a broad anticlinorium. Precambrian rocks were moved eastward over the Pennsylvanian rocks along steep reverse faults on the east flank of the rising mountains. The rocks within and immediately east of the zone of reverse faults were steeply tilted or overturned to form the series of hogbacks in which the district is located (Northrup, Sullwold, MacAlpin, and Rogers, 1946; Bachman, 1953). This narrow, upturned belt parallels the east flank of the mountains northward and as far south as Las Vegas.

The sedimentary rocks farther east were gently folded to form the Jarosa syncline and the Ocate anticline. The axes of these northtrending folds are 3 miles and 6 miles east of Coyote Creek.

Basalt flows belonging to two periods of activity in late Tertiary or early Quaternary time covered all older rocks. The older flows originally covered most of the district, but only scattered erosional remnants exist today.

\section{STRATIGRAPHY}

Four main groups of rocks in the Coyote district are: (1) igneous and metamorphic rocks of Precambrian age; (2) sandstone, arkose, siltstone, limestone, and shale of Pennsylvanian, Permian, and Triassic age; (3) basalt flows of late Tertiary or early Quaternary age; and (4) quaternary gravel and alluvium. Their distribution is shown in the general geologic map of the district in plate 24. A graphic section of the sedimentary rocks from Precambrian to the Benton formation of Cretaceous age is shown in figure 68. The rocks of Jurassic and Cretaceous age are included in figure 68 to provide comparison of the stratigraphy of the Coyote district with that of other uranium districts. Bachman (1953) gives descriptions of these units.

\section{PRECAMBRIAN ROCKS}

Precambrian rocks in fault contact with the Magdalena group crop out along the west side of the district. These Precambrian rocks are chiefly quartzite, containing minor amounts of pegmatite, schist, and gneiss. Coarse-grained pink granite, diabase, diorite, amphibolite, pegmatite, and quartz veins are common in the Precambrian areas of northern New Mexico and Colorado. Fragments of many of these rocks are found in the Pennsylvanian and Permian sedimentary rocks. 


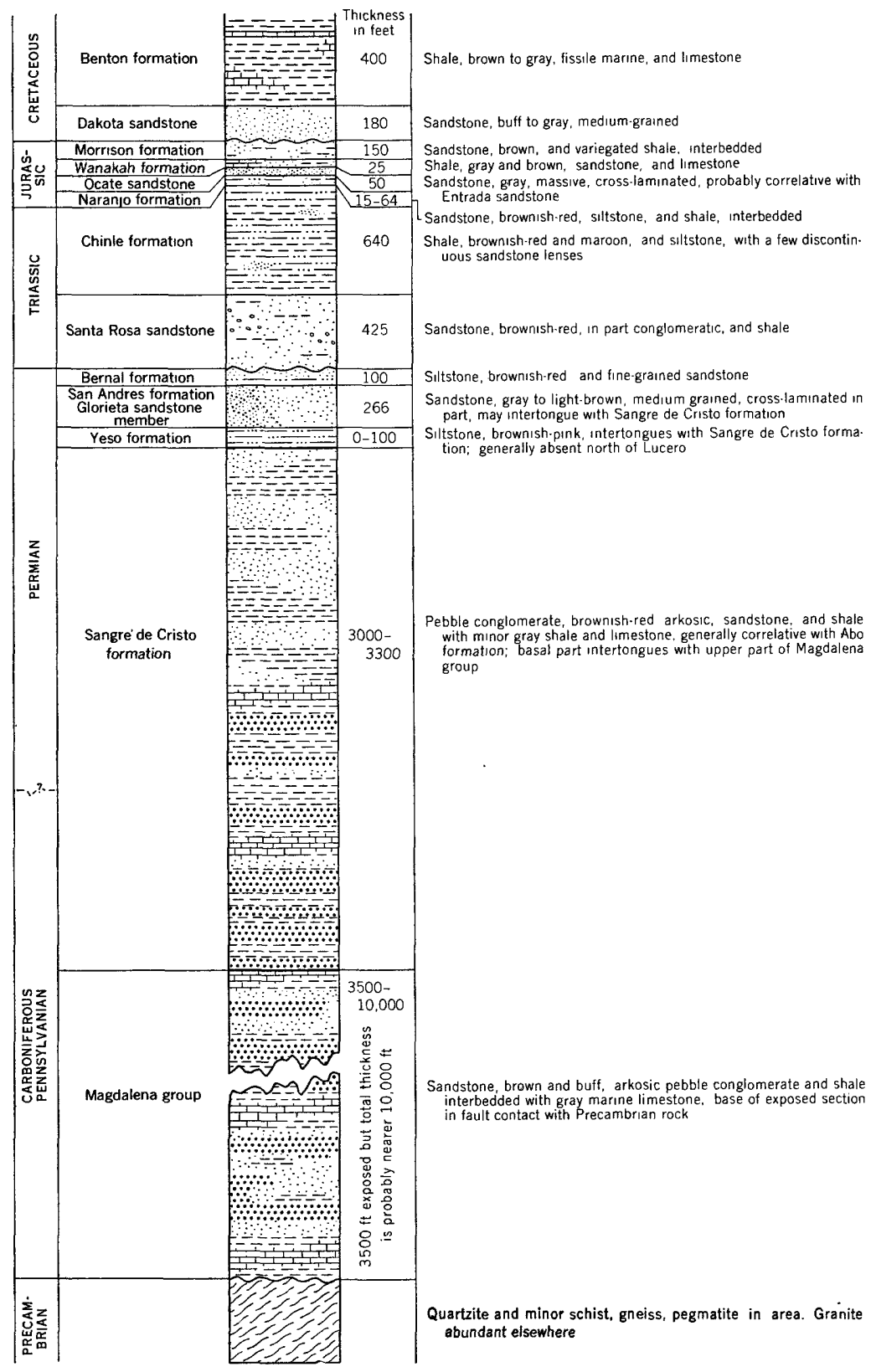

FradRe 68.-Generalized columnar section of the sedimentary rocks of the Coyote district and surrounding region. 


\section{MAGDALENA GROUP}

\section{DE9CRIPTION}

A regional angular unconformity separates the Precambrian rocks from the Magdalena group of Pennsylvanian age. The Magdalena group 12 miles west of Guadalupita near Chacon consists of four units (Bachman, 1953). The basal unit, 3,800 feet thick, is equivalent to the Sandia formation and consists of sandstone and arkose, containing minor amounts of shale and limestone. The next higher unit, about 1,000 feet thick, is bituminous and slightly calcareous friable and fissile shale. The third unit consists of 2,800 feet of interbedded arkose, sandstone, shale, and limestone. The upper unit, 3,000 feet thick, consists of brownish to reddish-brown sandstone, arkose, and shale interbedded with gray marine limestone.

The section at Chacon is more than 10,000 feet thick and is dominantly marine. Only about 3,500 feet of the upper part of the Magdalena group is exposed in the Coyote district, however. Bachman (1953) believes a large part of the Magdalena group is cut out by reverse faults along the west side of the district (pl. 24).

The marine sedimentary rocks of the Magdalena group in the Coyote district are yellow-brown, buff, or gray in contrast to the predominantly reddish continental sedimentary rocks of the Sangre de Cristo formation. On a regional scale the marine sedimentary rocks of the Magdalena group grade laterally and vertically into red beds of the Sangre de Cristo formation (Northrup and others, 1946). K. G. Brill (1952, p. 821-823, 826-827), G. O. Bachman (1953), and C. B. Read and G. H. Wood (1947, p. 223-227) give further details on the regional stratigraphic relationships of both Pennsylvanian and Permian formations.

The contact of the Magdalena group and the overlying Sangre de Cristo formation in the district was arbitrarily placed at the change from gray or brown to red about 140 feet above a 25 -foot fossiliferous limestone bed. In other parts of New Mexico the upper contact of the Magdalena group is at the top of the highest marine limestone bed, but in this area several thin marine limestone beds occur in the lower 950 feet of a red-bed sequence that is indistinguishable from the Sangre de Cristo formation.

\section{rossins}

The 25-foot-thick limestone bed 140 feet below the contact contains abundant marine fossils, including algae(?), sponges, corals, crinoids, bryozoans, brachiopods, and mollusks. The fossils listed here were identified by members of the Geological Survey (written communications, 1954). 
Corals (identified by Helen Duncan)

Caninoid corals (Pseudozaphrentoides as interpreted by Moore and Jeffords)

Clisiophyllid corals (Neokoninckophyllum as interpreted by Moore and Jeffords)

Bryozoans (identified by Helen Duncan)

Fistulipora sp., massive form

Polypora sp.

Rhabdomesonsp.

Brachiopods (identified by Mackenzie Gordon, Jr.)

Meekella striatocostata (Cox)

Dictyoclostus sp.

Linoproductus cf. L. platyumbonus. Dunbar and Condra

Echinoconchus semipunctatus (Shepard), var.

Neospirifer cf. $N$. latus. Dunbar and Condra

Composita subtilita (Hall)

Composita? sp.

Mollusks (identified by Mackenzie Gordon, Jr.)

Pteria sp.

Allorisma sp.

Worthenia sp. (identified by Ellis Yochelson and Brookes Knight)

The over-all affinities indicate about middle Pennsylvanian or at least post-Morrow age (Mackenzie Gordon, Jr., and Helen Duncan). The range of the species is little known in New Mexico but there is no evidence of a late Pennsylvanian or Permian age.

The species to which Linoproductus and Neospirifer are compared are confined to the Kansas City and Lansing groups of the Missouri series in Kansas and Nebraska, according to Dunbar and Condra (Mackenzie Gordon, Jr.). The corals resemble species from the upper Marble Falls limestone and Smithwick shale (post-Morrow lower Pennsylvania) of Texas more than other described species (Helen Duncan). A scale tree, probably Lepidodendron, found in arkose in the upper part of the Magdalena group indicates that marine conditions were not universal.

\section{SANGRE DE CRISTO FORMATION}

The Sangre de Cristo formation of Pennsylvanian and Permian(?) age overlies the Magdalena group with apparent conformity in the Coyote district, but it interfingers with the upper part of the Magdalena group on a regional scale.

\section{DESCRIPTION}

In the Guadalupita area, the Sangre de Cristo formation is between 3,000 and 3,200 feet thick (fig. 69) ; along the Mora River 6 miles south 


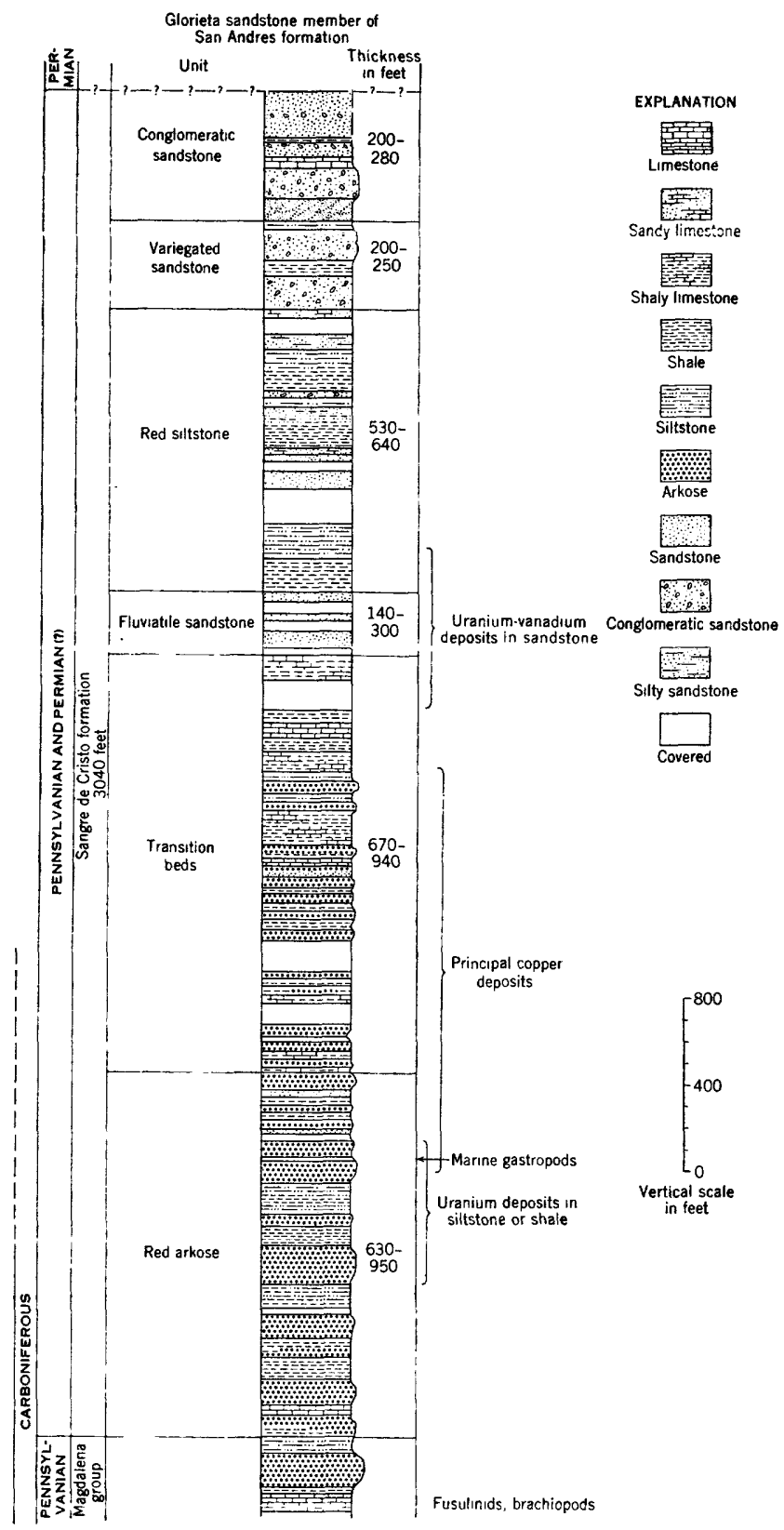

Figure 69.-Composite columnar section of the Sangre de Cristo formation, Coyote district, Mora County, N. Mex.

of the district, the thickness is 3,300 feet (Bachman, 1953). The formation is divided into six units for convenience of description. These units are not shown on the large-scale maps because of the uncertainty in tracing individual lenticular beds across faults, covered areas, and 


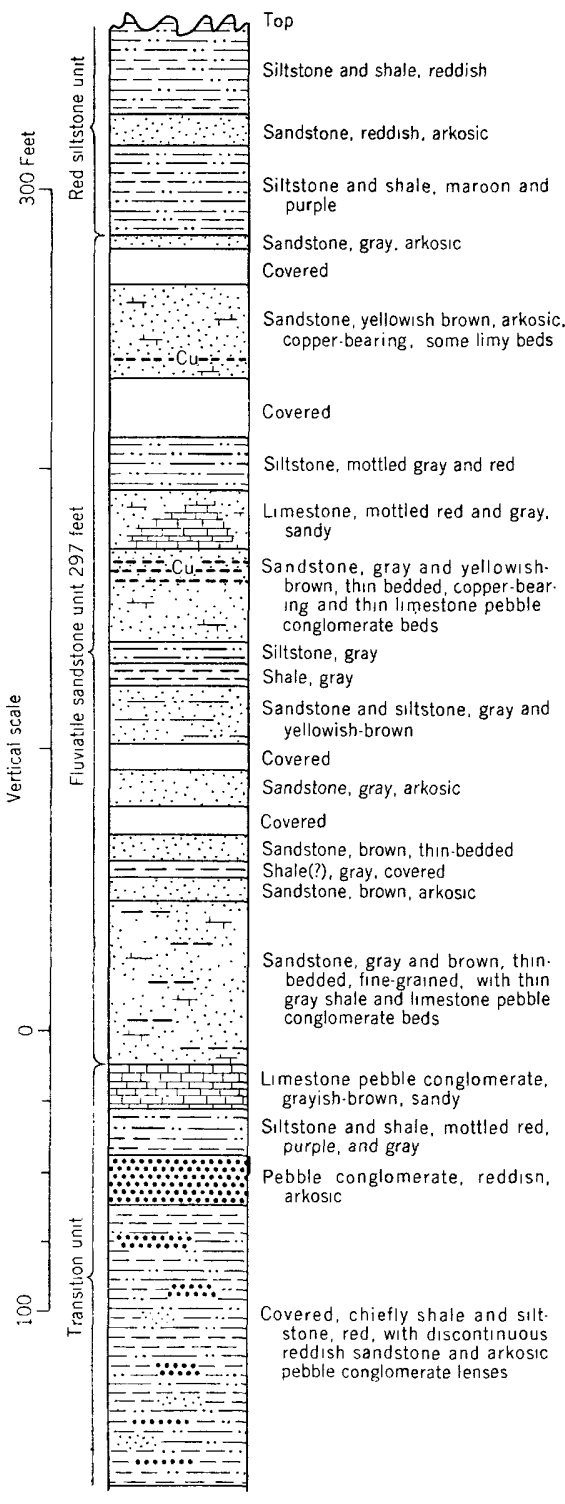

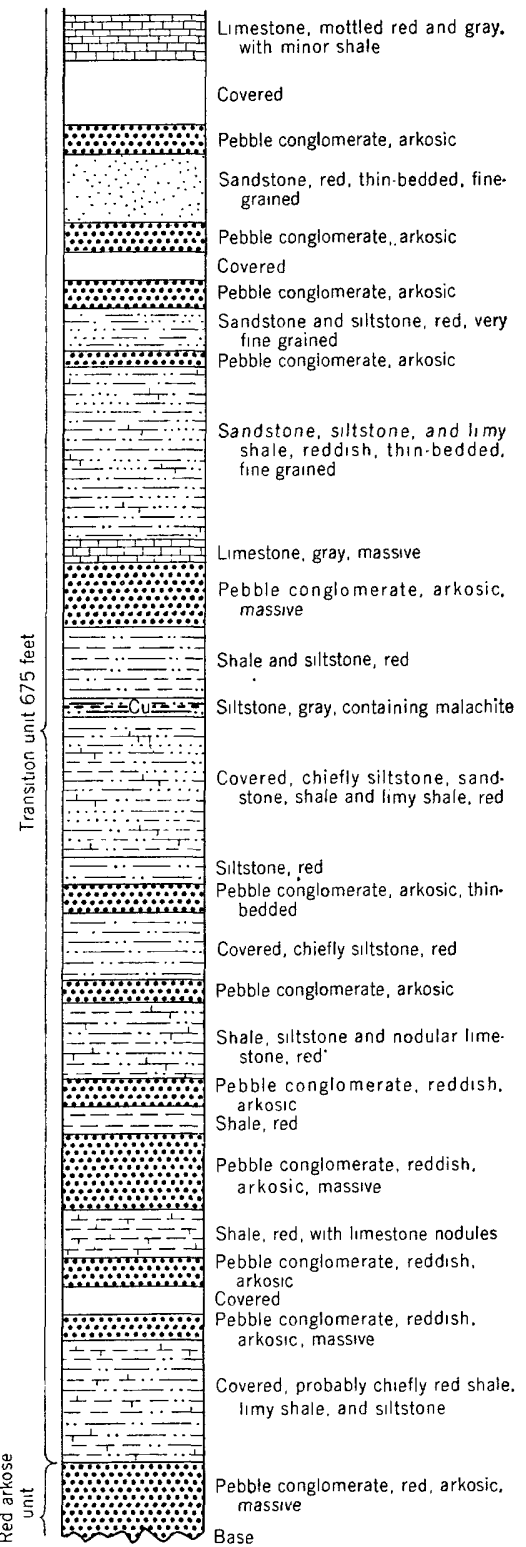

FIGURE 70.-Columanar section of the main ore-bearing interval, Sangre de Cristo formation, area $D$.

areas not mapped in detail. A lithologic description of these units is given in table 1.

A detailed columnar section of the middle, or principal ore-bearing part, of the formation in area $D$ is given in figure 70. Further details of the lithology are shown on the large-scale geologic maps of areas $A$ to $C$ and $E$ to $I$, plates 25 through 32 . Two notable characteristics 
of the Sangre de Cristo formation shown on these maps are: (1) the many abrupt facies changes and (2) the small stagnant basins in which the gray and black shale beds were deposited. No bed extends the length of the district.

TABLE 1.-Description of the Sangre de Cristo formation

\section{Conglomeratic sandstone unit}

Thickness

(feet)

200-280

Sandstone, reddish gray, friable, massive and crossbedded, conglomeratic, with abundant well-rounded sand grains and chert and quartzite pebbles up to 3 inches in diameter. Interbedded with some mottled silty limestone and shale. Sandstone in upper half weathers to smooth, rounded rim. May include beds equivalent to Yeso formation and lower part of the Glorieta sandstone member of San Andres formation. Unmineralized.

Variegated sandstone unit

$200 \div 250$

Sandstone, gray, brown, and red ; medium- to coarse-grained, poorly sorted, arkosic, fluviatile. Interbedded with thin red shale, siltstone, and limestone. Many cut-and-fill structures and abundant fragments of limestone, quartz, quartzite, siltstone, greenstone, and vein quartz. Unmineralized.

Red siltstone unit

Shale and siltstone, predominantly red, maroon, or chocolate; limy or micaceous. Many thin beds of silty limestone and fine-grained, red, silty sandstone. Some silicified wood at base. Unmineralized.

Fluviatile sandstone unit

Sandstone, gray or brown, well-sorted, thin-bedded, cross-bedded, or massive, fine- to medium-grained. Interbedded with thin gray shale, limy shale, and limestone pebble conglomerate. Sand-filled scours and channels common. Beds lenticular; some beds, limy; poorly sorted, coarse, with abundant clay fragments, carbonized wood, and foreign rock fragments. Uranium, vanadium, and copper deposits (pls. 26 and 29).

Transition unit

Arkosic pebble conglomerate, reddish gray, massive, alternating with red, gray, or black, micaceous, carbonaceous, or limy shale and siltstone. Many thin limestone and limy shale beds in upper part. Carbonized or silicified wood locally abundant. Copper in local gray or black lenses at 10 horizons; some uranium (pls. 27 and 31 ).

Red arkose unit

Arkosic pebble conglomerate, reddish gray, 20 to 80 feet thick. Coarse, massive beds alternating with red, chocolate, or maroon, shale, limy shale, and micaceous siltstone. Few thin limestone beds, some fossiliferous and marine. Arkose contains abundant angular quartz, feldspar, and granite fragments up to $3 / 4$ inch in diameter, carbonized, silicified or calcitized wood and magnetite seams along the bedding. A thin gray micaceous uraniferous shale or siltsone bed in areas $A$ and $C$. Some copper-bearing carbonaceous shale (pls. 25 and 32 ). 
FOSSILS

Carbonized plant remains and fossil wood replaced by silica, calcite, chalcocite, or, rarely, pink barite are the most common and widely distributed fossils in the Sangre de Cristo formation. Especially well-preserved plant remains are found in pit G2 and in the uraniferous siltstone in area $A$.

A Permian(?) conifer, Lebachia, was reported by Zeller and Baltz $(1954$, p. 3) in the northernmost pit in area $C$, and Calamites and Lepidodendron have been tentatively identified by the writers.

Some fossiliferous marine limestone or siltstone occurs in the lower 950 feet of the formation. Brachiopods, pelecypods, crinoids, nautiloids, and gastropods, chiefly bellerophontids, have been collected. The marine fossils have been identified by members of the Geological Survey as follows:

TABLE 2.-Marine fossils of the Sangre de Cristo formation

Fossil

Linoproductus sp

Myalina sp

Pelecypods indet

Hypselentoma? sp

Bellerophontid gastropods indet

Pseudorthoceras of. $P$. knoxense (McChesney)
Locality

i12 (pl. 32) .... BZ-21 (pl. 24) -

i6 (pl. 32)

i6 (pl. 32)

i6 (pl. 32)

i6 (pl. 32)

i6 (pl. 32)
Identified by

Mackenzie Gordon, Jr.

S. A. Northrup

E. L. Yochelson

Do.

Do.

Mackenzie Gordon, Jr. Do.

Some of the copper-bearing shale either contains marine fossils or is overlain by fossiliferous limestone. The genus Hypselentoma is known only from the upper Pennsylvanian in the mid-continent region (Yochelson, written communication, 1954). If the range is the same in the Coyote district, at least the lower 950 feet of the formation is Pennsylvanian.

\section{CONDITIONS OF DEPOSITION}

Most of the Sangre de Cristo formation appears to be first-cycle sediments deposited in a deltaic, flood plain, or fluviatile environment near the source areas, which were high during the deposition of the lower units of the formation. Thin, marine limestone beds show that the basin of deposition was intermittently below sea level. The thickness and grain size of the coarser beds decreases upward, and the proportion of limestone or shale increased as the relief of the source areas became lower. The lower two units show a cyclic sequence of sediments; these have been called cyclothems of the piedmont type.

Locally, especially during deposition of the transition unit, swamps, lagoons, and stagnant ponds existed on the flood plain. According to Yochelson (written communication, 1954), the abundance of Hypselentoma(?) in a limestone bed at pit i6 and the presence of 
very few other marine fossils could indicate a shallow-water environment with restricted circulation of sea water.

The sandstone of the fluviatile sandstone unit (table 1) was apparently deposited in the channels of meandering streams on a flood plain. The coarse, poorly sorted, conglomeratic sandstone beds above. the red siltstone unit may indicate renewed uplift or a new source area. The uppermost unit appears to be a series of beach deposits on an area. being gradually inundated.

\section{YESO FORMATION}

The Yeso formation of Permian age pinches out near Lucero and is not present in the mapped area. It consists of brownish-pink siltstone that is lighter and finer than the Sangre de Cristo formation. The description of this and succeeding units is from Bachman (1953).

\section{SAN ANDRES FORMATION (GLORIETA SANDSTONE MEMBER)}

The Glorieta sandstone member of the San Andres formation of Permian age is the only member of the formation exposed in the district. The member consists of about 266 feet of gray to light-brown, medium-grained sandstone. It forms the prominent cliff along the east side of the district.

\section{BERNAL FORMATION}

The Bernal formation of Permian age consists of interbedded brownish-red siltstone and fine-grained sandstone about 100 feet thick: A disconformity separates the Bernal formation and the Glorieta sandstone member of the San Andres formation.

\section{SANTA ROSA SANDSTONE}

The Santa Rosa sandstone of Late Triassic age is exposed in the southeast corner of the area (pl. 24), lying disconformably on the Bernal formation. It consists of 425 feet of brownish-red shale and sandstone. The units above the Santa Rosa sandstone are not exposed in the district and are not described although they are included in figure 68.

\section{QUATHRNARY (?) BASALT}

Five volcanic centers are present a short distance northeast of the Coyote district. The nearest is less than a mile east of the north end of the district. Two ages of extensive basalt flows (Bachman, 1953) cover large areas along the east and north sides of the district. The older flows originally covered the Coyote district but only remnants now exist as mesa cappings on vertical beds of older rocks (pl. 24). The five volcanic plugs and the flow rocks (Bachman, 1953) in the Ocate area are products of the later period of eruption. No evidence has been found of a genetic relationship between the basalt and the mineral deposits. 


\section{STRUCTURE}

The Coyote district is in a Laramide( ?) belt of deformation along the east flank of the Sangre de Cristo Range. The rocks east of the district are warped into gentle folds; the rocks west of Coyote Creek are turned up sharply along a north-south axis. In the mineral belt the dips are steep to overturned and farther west the beds are overturned and cut by steep reverse faults. Bedding attitudes within the mineral belt indicate that the forces of deformation were torsional, increasing in intensity northward. The strike changes progressively northward from N. $20^{\circ}$ W. to N. $20^{\circ}$ E. (pl. 24). At the south end the beds dip $80^{\circ}$ E. Northward the dip passes through the vertical, and the overturned beds in the northern part dip $50^{\circ}-75^{\circ} \mathrm{W}$. The degree of overturn increases westward throughout most of the district.

Several high-angle reverse faults were mapped by Bachman (1953) in Precambrian and Pennsylvanian rocks along the west side of the district. The Precambrian rocks have been thrust eastward several thousand feet over Pennsylvanian rocks, cutting out a large part of the Magdalena group.

Several northwest-striking oblique faults with lateral displacement of less than 300 feet were mapped in areas $A, E$, and $F$ (pls. 25, $28,29)$. Several minor northeast-striking oblique faults were mapped in area $H$ (pl. 31). Some faults clearly offset the ore zones; however, the lack of marker beds makes correlation across the faults difficult. The arkose is sheared and bleached in areas of closely spaced faulting. Although several ore deposits are near major faults there is no evidence of mineral deposition along the faults (pls. 28 and 29).

\section{MINERAL DEPOSITS}

The mineral deposits of the Coyote district (pl. 24) are confined to lenticular beds of gray, brown, black, or pink sedimentary rocks in the lower 2,000 feet of the Sangre de Cristo formation. Copper and uranium minerals have not been found in the red, chocolatecolored, or maroon rocks that make up most of the formation.

The ore minerals are sparsely distributed, and individual deposits are small. Copper deposits typically contain negligible amounts of uranium and the uranium deposits contain small but variable amounts of copper.

Uranium deposits occur in sandstone and micaceous siltstone; copper deposits occur in several rock types, but most of the ore bodies are in black carbonaceous shale. The fluviatile sandstone unit (table 1) contains most of the uranium deposits. Most of the copper deposits are in lower units, particularly the transition unit.

The location of the mineral deposits and samples are shown on the large-scale maps, plates 25-32. The analytical data for samples 
from each area are given in tables 3-9. The location of the scattered samples outside these areas are shown in plate 24 and analyses are given in table 10 .

\section{COPPER DEPOSTTS}

\section{DESCRIPTION}

Copper deposits occur in four types of rock: (1) carbonaceous shale or siltstone, (2) arkosic pebble conglomerate, (3) micaceous sandstone, and (4) nodular limy shale or limestone. The deposits are richest and most abundant in black, carbonaceous shale lenses and in adjacent parts of gray shale or arkosic pebble conglomerate beds. Sandstone normally contains low-grade disseminated deposits, and copper deposits in limestone are rare and unimportant.

TABLE 3.-Analytical data for samples from area $A$ ( $p l .25)$

[Radioativity analyses by S. P. Furman and E. S. Fennely. Chemical analyses by H. E. Bivens, R. F. Dufour, E. C. Mallory, C. E. Thompson, D. C. Stockwell, J. H. McCarthy, J. F. Wahlberg, and W. D. Goss]

\begin{tabular}{|c|c|c|c|c|c|c|}
\hline \multirow{2}{*}{$\begin{array}{c}\text { Field } \\
\text { no. }\end{array}$} & \multirow{2}{*}{$\underset{\text { (ft.) }}{\text { Width }}$} & \multicolumn{3}{|c|}{ Percent } & \multirow{2}{*}{$\begin{array}{c}\mathrm{Se} \\
(\mathrm{ppm})\end{array}$} & \multirow{2}{*}{ Lithologic description } \\
\hline & & $\mathrm{eU}$ & $\mathrm{U}$ & $\mathrm{Ou}$ & & \\
\hline 92 & 2.0 & 0.007 & 0.005 & 5. 07 & & Green sandstone and shale. \\
\hline 03 & & & & & & Black carbonaceous shale. \\
\hline 94 & $-1+8$ & $\ldots$ & & $\ldots$ & & Altered limonitic sandstone. \\
\hline 194 & 2. 7 & .004 & $\ldots$ & .13 & & Buff and gray shale. \\
\hline 195 & 3. 2 & .007 & .004 & .07 & & Buff siltstone, brown shale. \\
\hline 196 & .6 & .013 & .008 & .10 & & Brown siltstone and gray shale. \\
\hline 197 & 2. 8 & .004 & {$[-\ldots$} & $\ldots$ & & Buff sandstone and gray siltstone. \\
\hline 198 & 2. 3 & .008 & .003 & .15 & & Brown siltstone with plant remains. \\
\hline 199 & 2. 5 & .011 & .006 & .10 & & Gray-brown siltstone. \\
\hline 200 & 1.4 & .004 & $\ldots$ & .10 & & Yellow-brown siltstone and shale. \\
\hline 201 & 1. 0 & .044 & .029 & .15 & & Yellow-brown micaceous siltstone. \\
\hline 202 & 2.4 & .005 & $<.005$ & .10 & & Yellow-brown shale and siltstone. \\
\hline 203 & 2. 4 & .011 & .006 & .10 & & $\begin{array}{l}\text { Yellow-brown siltstone with plant } \\
\text { remains. }\end{array}$ \\
\hline 204 & 3. 0 & .010 & .005 & .20 & & Do. \\
\hline 205 & 1.5 & .008 & .004 & .20 & & Do. \\
\hline 206 & 2. 7 & .011 & .007 & .15 & & Yellow-brown shale and siltstone. \\
\hline 207 & 2.4 & .008 &.- & .07 & & Olive-brown siltstone. \\
\hline 208 & 2.1 & .012 & .005 & $\ldots$ & & Do. \\
\hline 209 & 2. 1 & .015 & (-.... & 15 & 2 & Gray-green siltstone and shale. \\
\hline 210 & 3. 5 & .009 & $\ldots \ldots$ & .10 & & Dark gray-green siltstone with shale. \\
\hline 211 & 3. 5 & .009 & & .07 & -1 & $\begin{array}{l}\text { Gray-green and olive-brown shale } \\
\text { and siltstone. }\end{array}$ \\
\hline 212 & 2. 8 & .002 & & .07 & & Gray-green shale and siltstone. \\
\hline 213 & 9 & .003 & & .10 & & Gray shale. \\
\hline 214 & 2. 0 & .019 & & .10 & 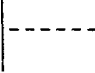 & $\begin{array}{l}\text { Gray shale and yellow-brown silt- } \\
\text { stone with carbon seams. }\end{array}$ \\
\hline
\end{tabular}


TABLE 3.-Analytical data for samples from area $A$ (pl. 25)-Continued

\begin{tabular}{|c|c|c|c|c|c|c|}
\hline \multirow{2}{*}{$\begin{array}{c}\text { Field } \\
\text { no. }\end{array}$} & \multirow{2}{*}{$\begin{array}{l}\text { Width } \\
\text { (ft.) }\end{array}$} & \multicolumn{3}{|c|}{ Percent } & \multirow{2}{*}{$\begin{array}{c}\mathrm{Se} \\
(\mathrm{ppm})\end{array}$} & \multirow{2}{*}{ Lithologic description } \\
\hline & & $\mathrm{eU}$ & $\mathrm{u}$ & $\mathrm{Cu}$ & & \\
\hline 215 & 1. 6 & 0.004 & ... & 0.07 & 2 & Gray and black silty sandstone. \\
\hline 216 & 1. 4 & .019 & 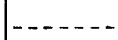 & .15 & & Yellow-brown siltstone and shale. \\
\hline 217 & 1. 9 & .006 & $\ldots \ldots$ & .10 & & Do. \\
\hline 219 & .9 & .004 & 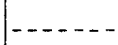 & .03 & & Do. \\
\hline 220 & 1. 8 & .004 & $\ldots$ & .06 & & Gray-green and olive shale. \\
\hline 221 & 2. 7 & .007 & & .05 & 5 & Yellow-brown siltstone and shale. \\
\hline 222 & .8 & .006 & $\cdots$ & .02 & & Gray micaceous siltstone and shale. \\
\hline 223 & $\ldots$ & $-\ldots$ & & $\ldots$ & & Chalcocite nodules. \\
\hline 224 & $\ldots$ & -1 & & -- & & Fossiliferous limestone. \\
\hline 225 & 1. 6 & .006 & $-\ldots-$ &.- & & Gray-green shale. \\
\hline 226 & 1. 6 & .022 & 021 & & & Gray-green shale with carbon seams. \\
\hline 231 & 1. 2 & .016 & .011 & & & $\begin{array}{l}\text { Siltstone with calcite, quartz, plagio- } \\
\text { clase, nontronite, and kaolinite. }\end{array}$ \\
\hline 233 & .9 & .013 & .007 & 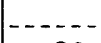 & & Gray-green micaceous siltstone. \\
\hline 234 & .7 & .018 & .012 & .36 & & Black siltstone with carbon seams. \\
\hline 235 & 1.2 & .021 & .014 & . & & $\begin{array}{l}\text { Gray-green shale and yellow-brown } \\
\text { sandstone. }\end{array}$ \\
\hline 236 & .9 & .011 & .008 & & & $\begin{array}{l}\text { Dark gray-green siltstone with car- } \\
\text { bon. }\end{array}$ \\
\hline 237 & 1. 7 & .012 & .009 & & & Do. \\
\hline 238 & 1.6 & .026 & .015 & .17 & & Yellow-green micaceous sandstone. \\
\hline 239 & 2.6 & .006 & $-\ldots$ & $\ldots-$ & & Micaceous sandstone. \\
\hline 240 & 1. 0 & .020 & .015 & .29 & & Yellow-green micaceous sandstone. \\
\hline 241 & 3. 3 & .011 & .009 & $\cdots$ & & Do. \\
\hline 242 & 2. 1 & .006 & 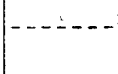 &. .16 & & $\begin{array}{l}\text { Dark-green micaceous shale, yellow- } \\
\text { brown sandstone. }\end{array}$ \\
\hline 243 & 1. 8 & .012 & .007 & .20 & & $\begin{array}{l}\text { Gray-green shale and carbon seams, } \\
\text { yellow-brown sandstone. }\end{array}$ \\
\hline 244 & .8 & .008 & .002 &, 54 & & Dark-green and black shale. \\
\hline 245 & $\therefore .8$ & .012 & .007 & $-\ldots$ & & Fine-grained sandstone, \\
\hline 400 & 2. 0 & .002 & & .29 & & Blue-gray shale. \\
\hline 401 & 1. 0 & .002 & & .60 & & Blue-gray arkosic sandstone. \\
\hline 402 & 2.0 & .005 & & 3. 92 & & Black shale and arkosic sandstone. \\
\hline 403 & 6. 0 & .004 & $\ldots$ & 1. 75 & & Composite of 401 and 402 . \\
\hline 404 & 3. 5 & .002 & $\ldots . .$. & .14 & $-\ldots$ & Gray-green shale. \\
\hline 405 & 2. 5 & .002 & $\ldots . .$. & .05 & & Gray-green micaceous siltstone. \\
\hline 412 & 3. 3 & .004 & $\ldots-$ & .18 & -- & Dark gray-green micaceous shale. \\
\hline 228 & 2. 0 & .008 & $-\cdots$ & $-\ldots$ & $-\ldots$ & Yellow-brown micaceous siltstone. \\
\hline
\end{tabular}




\section{COPPER AND URANIUM DEPOSITS, COYOTE DISTRICT, N. MWX. 359}

TABLE 4.-Analytical data for samples from area $B$ (pl. 26)

[Radioactivity analyses by S. P. Furman. Chemical analyses by E. C. Mallory, J, F. Wahlberg, Wayne Mountjoy, S. P. Schuch, H. E. Crowe; W. D. Goss, and J. H. McCarthy]

\begin{tabular}{|c|c|c|c|c|c|c|}
\hline \multirow{2}{*}{$\begin{array}{c}\text { Field } \\
\text { no. }\end{array}$} & \multirow{2}{*}{$\begin{array}{l}\text { Width } \\
\text { (feet) }\end{array}$} & \multicolumn{4}{|c|}{ Percent } & \multirow{2}{*}{ Lithologic description } \\
\hline & & eU & U & $\mathrm{Cu}$ & $\mathrm{V}_{2} \mathrm{O}_{6}$ & \\
\hline 247 & 1.0 & 0.051 & 0.036 & 0.79 & 一 & $\begin{array}{l}\text { Coarse gray-brown arkosic sandstone } \\
\text { with malachite. }\end{array}$ \\
\hline 248 & .6 & .058 & .049 & 1. 37 & - & $\begin{array}{l}\text { Brown and yellow-brown, fine-grained, } \\
\text { thin-bedded arkosic sandstone with } \\
\text { malachite. }\end{array}$ \\
\hline 249 & .6 & .061 & .041 & 1. 22 & - & Same as 248 with sulfides. \\
\hline 251 & - & .11 & .10 & .01 & - & $\begin{array}{l}\text { Gray-brown arkosic sandstone with } \\
\text { chalcopyrite and calcite cement. }\end{array}$ \\
\hline 330 & - & .052 & .044 & .77 & 0.32 & Carbonized-ocherous log. \\
\hline 331 & - & - & - & $\longrightarrow$ & - & $\begin{array}{l}\text { Pink arkosic sandstone with chalco- } \\
\text { pyrite. }\end{array}$ \\
\hline 332 & .8 & .022 & .017 & .02 & $<.1$ & Pink-gray arkosic sandstone. \\
\hline 333 & 1. 2 & .043 & .033 & .62 & .12 & Gray arkosic sandstone. \\
\hline 334 & 1. 2 & .048 & .024 & 1.66 & .15 & Black and brown sandstone. \\
\hline 335 & .4 & .030 & .033 & .25 & $<.1$ & Arkosic sandstone. \\
\hline 336 & .5 & .024 & .018 & .63 & $<.1$ & Pink feldspathic sandstone. \\
\hline 352 & .5 & .26 & .29 & .47 & .22 & $\begin{array}{l}\text { Pink arkosic sandstone with chalco- } \\
\text { pyrite and metatyuydmunite. }\end{array}$ \\
\hline
\end{tabular}


TABLE 5.-Analytical data for samples from area $C$ (pl. 27)

[Radioactivity analyses by S. P. Furman and E. J. Fennelly. Chemical analyses by D. L. Skinner, J. H. McCarthy, W. N. Niles, Wayne Mountjoy, J. P. Schuch, H. E. Bivens, R. F. Dufour, E. C. Mallory, C. E. Thompson, D. L. Stoekwell, and J. F. Mallory]

\begin{tabular}{|c|c|c|c|c|c|c|c|}
\hline \multirow{2}{*}{$\begin{array}{l}\text { Field } \\
\text { no. }\end{array}$} & \multirow{2}{*}{$\begin{array}{l}\text { Width } \\
\text { (feet) }\end{array}$} & \multicolumn{4}{|c|}{ Percent } & \multirow{2}{*}{$\begin{array}{c}\mathrm{Se} \\
\text { (ppm) }\end{array}$} & \multirow{2}{*}{ Lithologic description } \\
\hline & & $\mathrm{eU}$ & U & $\mathrm{Cu}$ & $\mathrm{V}_{2} \mathrm{O}_{6}$ & & \\
\hline 58 & & & & & & & Chalcocite nodules. \\
\hline 59 & & 0.004 & 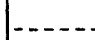 & $\cdots$ & & & Gray-green shale. \\
\hline 60 & $\ldots$ & .004 & $-\ldots$ & 0.15 & & & Do. \\
\hline 65 & $\ldots-\ldots$ & .006 & 0.004 & .88 & $<0.1$ & 2 & $\begin{array}{l}\text { Dark gray micaceous silt- } \\
\text { stone. }\end{array}$ \\
\hline 66 & & . & & & & & Black carbonaceous shale. \\
\hline 67 & & $\ldots . .$. & -..... & $\ldots \ldots$ & & & Wood replaced by chalcocite. \\
\hline 68 & -- & --- & - & $7-1$ & & & Chalcocite nodules. \\
\hline 69 & & & & $\ldots$ & & & Large chalcocite nodules. \\
\hline 70 & 3. 4 & .008 & .005 & 2. 38 & $<$. & 2 & Black carbonaceous shale. \\
\hline 71 & 2. 0 & .005 & .003 & 2. 14 & <. & 2 & Gray-green shale. \\
\hline 72 & $\ldots$ & $-\ldots-\ldots$ & $\ldots$ & $\ldots$ & & & Chalcocite nodules. \\
\hline 73 & $\ldots$ & -1 & $\ldots \ldots$ & -1 & & & Do. \\
\hline 337 & 4.0 & .01 & .004 & 2. 63 & & & $\begin{array}{l}\text { Black shale with chalcocite } \\
\text { nodules. }\end{array}$ \\
\hline 338 & 1.0 & .008 & .002 & 1.01 & & & Do. \\
\hline 339 & 1.3 & .005 & .001 & 1. 91 & & & Do. \\
\hline 340 & 1.7 & .004 & .001 & 1.61 & & & Do. \\
\hline 341 & 1. 7 & .005 & .001 & 1. 94 & & & Do. \\
\hline 342 & .6 & .007 & .004 & 1.04 & $\cdots$ & & Do. \\
\hline 414 & 2. 1 & .016 & .009 & .09 & C. 1 & & Cross-bedded silty sandstone. \\
\hline 415 & .6 & $\ldots-\ldots$ & $-\ldots$ & & & & $\begin{array}{l}\text { Yellow - brown micaceous } \\
\text { sandstone. }\end{array}$ \\
\hline 416 & 4. 1 & .019 & .012 & .08 & .17 & & Yellow sandstone. \\
\hline 417 & 2. 1 & .034 & .016 & .56 & & & $\begin{array}{l}\text { Gray-green shale and yellow } \\
\text { sandstone. }\end{array}$ \\
\hline 418 & 1.5 & .010 & .006 & .02 & & & Micaceous sandstone. \\
\hline 419 & .9 & 023 & .011 & .20 & & & Do. \\
\hline 420 & .6 & .003 & .002 & .05 & & & $\begin{array}{l}\text { Yellow - green micaceous } \\
\text { sandstone. }\end{array}$ \\
\hline BZ-1 & & .009 & .006 & 2. 21 & & & $\begin{array}{l}\text { Dark - gray carbonaceous } \\
\text { shale. }\end{array}$ \\
\hline BZ-2 & ---- & .007 & .007 & 1. 73 & & & $\begin{array}{l}\text { Arkosic sandstone with chal- } \\
\text { cocite and malachite. }\end{array}$ \\
\hline $\mathrm{BZ}-3$ & 2. 0 & .020 & .004 & 2. 51 & & & Black carbonaceous shale. \\
\hline $\mathrm{BZ}-4$ & $\ldots$ & .007 & .003 & 2. 72 & & $\ldots$ & $\begin{array}{l}\text { Black carbonaceous shale } \\
\text { with chalcocite nodules } \\
\text { and carbonized plant re- } \\
\text { mains. }\end{array}$ \\
\hline
\end{tabular}


TABLE 6.-Analytical data for samples from area $E$ (pl. 28)

IRadtractivity analyses by S. P. Furman and E. J. Fennelly. Chemical analyses by D. L. Skinner, J. H. MeCarthy, W. N. Niles, Wayne Mountjoy, J. P. Schuch, W. D. Goss, H. E. Bivens, R. F. Dufour, E. O. Mallory, and C. E. Thompson]

\begin{tabular}{|c|c|c|c|c|c|c|c|}
\hline \multirow{2}{*}{ Fleld no. } & \multirow{2}{*}{$\begin{array}{c}\text { Thick- } \\
\text { ness } \\
\text { (feet) }\end{array}$} & \multicolumn{4}{|c|}{ Percent } & \multirow{2}{*}{$\begin{array}{c}\text { Se } \\
\text { (ppm) }\end{array}$} & \multirow{2}{*}{ Lithologie description } \\
\hline & & $\mathrm{eU}$ & 0 & Ou & $\mathrm{V}_{2} \mathrm{O}_{6}$ & & \\
\hline 40 & & 0.008 & & 2. 74 & & & Gray carbonaceous shale. \\
\hline 41 & & .003 & & $\ldots$ & & & Black arkosic sandstone. \\
\hline 42 & 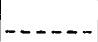 & $-\ldots$ & $\ldots .$. & $\ldots$ & & & Chalcocite in sandstone. \\
\hline 43 & $\ldots \ldots$ & .006 & $-\ldots$ & 4. 08 & & & Do. \\
\hline 44 & $\ldots \ldots$ & .005 & & $\ldots-$. & & & Arkosic sandstone. \\
\hline 45 & & $\ldots \ldots$ & & $\ldots \ldots$ & & & $\begin{array}{l}\text { Black arkosic sandstone } \\
\text { with malachite and } \\
\text { volborthite. }\end{array}$ \\
\hline 46 & & & & & & & Chalcocite nodules. \\
\hline 47 & 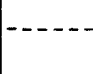 & .003 & - & $\ldots \ldots$ & & & $\begin{array}{l}\text { Green shale and sand- } \\
\text { stone. }\end{array}$ \\
\hline 48 & & .005 & $\ldots$ & 1. 46 & & & $\begin{array}{l}\text { Gray sandstone with } \\
\text { malachite and volbor- } \\
\text { thite. }\end{array}$ \\
\hline 91 & & & & & & & $\begin{array}{l}\text { Chalcocite and meta- } \\
\text { tyuyamunite in sand- } \\
\text { stone. }\end{array}$ \\
\hline 97 & 5. 5 & .004 & $-\ldots \ldots$ & .49 & $<0.1$ & $<2$ & Gray silty sandstone. \\
\hline 98 & 5. 0 & .002 & -..... & .15 & $<.1$ & $<2$ & $\begin{array}{l}\text { Yellow-brown silty } \\
\text { sandstone. }\end{array}$ \\
\hline 99 & 2.4 & .005 & 0.002 & .22 & $<.1$ & $<2$ & $\begin{array}{l}\text { Gray micaceous silty } \\
\text { sandstone. }\end{array}$ \\
\hline 100 & .5 & .010 & .009 & 4. 65 & .26 & 10 & $\begin{array}{l}\text { Green micaceous silt- } \\
\text { stone. }\end{array}$ \\
\hline 101 & .9 & .004 & $\ldots$ & .91 & .30 & $<2$ & Do. \\
\hline 102 & 1. 7 & .005 & .004 & 1. 34 & .18 & 3 & Do. \\
\hline 103 & 1. 1 & .011 & .009 & 5. 81 & $<.1$ & 3 & Do. \\
\hline 104 & & $\ldots . .$. & . & 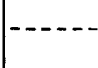 & & & $\begin{array}{l}\text { Argillized arkosic sand- } \\
\text { stone. }\end{array}$ \\
\hline $\mathrm{BZ}-7$ & & .014 & .010 & 4. 16 & & & Arkosic sandstone. \\
\hline BZ-8 & $\ldots$ & .065 & .048 & 6. 39 & --- & & $\begin{array}{l}\text { Arkosic sandstone with } \\
\text { metatyuyamunite. }\end{array}$ \\
\hline BZ-9 & & .039 & .018 & 14. 38 & 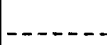 & -- & Do. \\
\hline $\mathrm{BZ}-10$ & $\ldots$ & .001 & .001 & $\ldots \ldots$ & 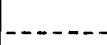 & -. & Barren olive clay shale. \\
\hline $\mathrm{BZ}-11$ & $\ldots$ & .001 & $<.001$ & .03 & . & . & Barren gray clay shale. \\
\hline BZ-12 & $\ldots$ & .011 & .008 & 3. 72 & - & $\ldots$ & $\begin{array}{l}\text { Shale and arkosic sand- } \\
\text { stone. }\end{array}$ \\
\hline$B Z-13$ & & .018 & .015 & 4. 65 & & & $\begin{array}{l}\text { Black carbonaceous } \\
\text { shale. }\end{array}$ \\
\hline BZ-14 & & & & & & & Radioactive coaly frag- \\
\hline & & & & & & & $\begin{array}{l}\text { ment may contain } \\
\text { uraninite(?). }\end{array}$ \\
\hline
\end{tabular}


TABLE 7.-Analytical data for samples from area $F$ (pl. 29)

IRadicactivity analyses by S. P. Furman and E. J. Fennelly. Chemical analyses by D. L. Skinner J. H. McCarthy, H. E. Bivens, R. F. Dufour, E. C. Mallory, C. E. Thompson, J. K. Wilson, D. L. Stockwell, W. D. Qoss, J. F. Wahlberg, and J. L. Stlverly]

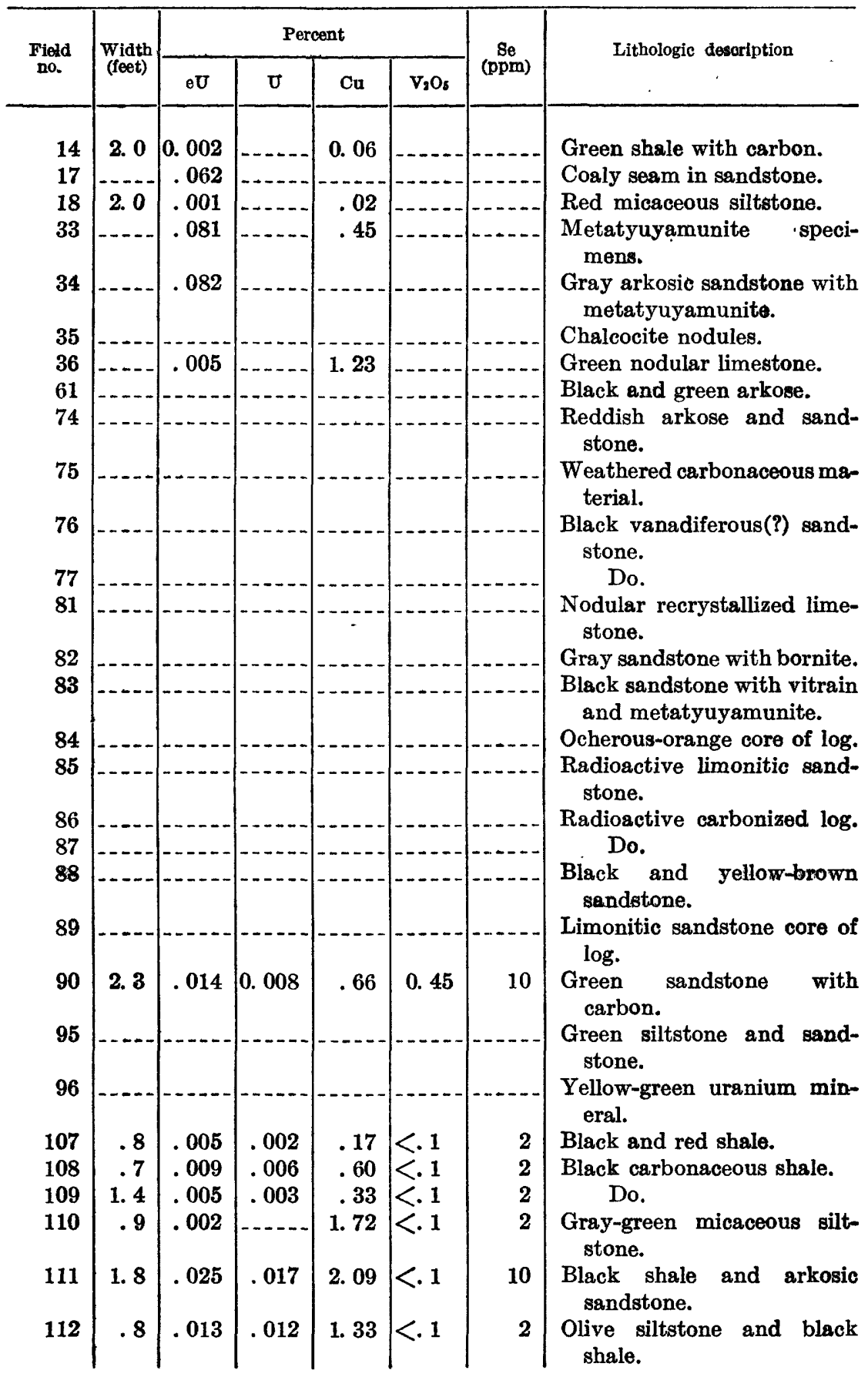


COPPER AND URANIUM DEPOSITS, COYOTE DISTRICT, N. MEX. 363

TABLE 7.-Analytical data for samples from area $F$ (pl. 29)-Continued

\begin{tabular}{|c|c|c|c|c|c|c|c|}
\hline \multirow{2}{*}{$\begin{array}{c}\text { Field } \\
\text { no. }\end{array}$} & \multirow{2}{*}{$\begin{array}{l}\text { Width } \\
\text { (feet) }\end{array}$} & \multicolumn{4}{|c|}{ Percent } & \multirow{2}{*}{$\underset{(\mathrm{ppm})}{\mathrm{Se}}$} & \multirow{2}{*}{ Lithologic description } \\
\hline & & $\mathrm{eU}$ & U & $\mathrm{Cu}$ & $\mathrm{V}_{2} \mathrm{O}_{5}$ & & \\
\hline 113 & & & & -1 & & & Chalcocite nodules. \\
\hline 114 & $\ldots$ & - - & $\ldots$ & $\ldots$ & & & Black shale. \\
\hline 116 & 3. 5 & 0.004 & & 0.06 & $<.1$ & $<2$ & Red, gray, and green shale. \\
\hline 176 & 3. 5 & .005 & 0.004 & .3 & & & Gray, black, and olive shale. \\
\hline 177 & 1. 4 & .007 & .005 & .58 & & & $\begin{array}{l}\text { Olive and gray-green silt- } \\
\text { stone. }\end{array}$ \\
\hline 178 & 3. 4 & .003 & .004 & & & & Olive, gray, and black shale. \\
\hline 179 & 1.1 & .005 & .004 & 2 & & & Olive and black shale. \\
\hline 186 & 2. 0 & .18 & .20 & 1. 27 & & & $\begin{array}{l}\text { Pink arkosic sandstone, mi- } \\
\text { caceous siltstone with } \\
\text { metatyuyamunite. }\end{array}$ \\
\hline 187 & 1. 8 & .063 & .042 & .89 & & & $\begin{array}{l}\text { Pink sandstone and black } \\
\text { shale. }\end{array}$ \\
\hline 188 & 1. 3 & .009 & .006 & .72 & & & Olive-brown and black shale. \\
\hline 343 & 2. 1 & .015 & .006 & 02 & 29 & & Arkosic sandstone. \\
\hline 344 & 1. 2 & .042 & .027 & .10 & .90 & & $\begin{array}{r}\text { Conglomeratic sandstone } \\
\text { with black clay fragments. }\end{array}$ \\
\hline 345 & 2. 0 & .075 & .038 & .17 & 1. 22 & & $\begin{array}{l}\text { Pink conglomeratic arkosic } \\
\text { sandstone. }\end{array}$ \\
\hline 346 & 2. 2 & .046 & .041 & .09 & 1. 36 & & $\begin{array}{l}\text { Gray-brown arkosic sand- } \\
\text { stone. }\end{array}$ \\
\hline 347 & 1. 3 & .038 & .024 & .09 & 32 & & Pink feldspathic sandstone. \\
\hline 349 & $\ldots$ & .20 & .18 & .20 & 2. 07 & & $\begin{array}{l}\text { Selected high grade, abun- } \\
\text { dant metatyuyamunite. }\end{array}$ \\
\hline 350 & 1.0 & .038 & .033 & .02 & $<.1$ & & Pink arkosic sandstone. \\
\hline 351 & 1. 2 & .044 & .036 & .02 & $<.1$ & 3 & Do. \\
\hline 353 & 1. 3 & .068 & .019 & 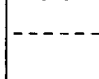 & & & $\begin{array}{l}\text { Pink arkosic micaceous sand- } \\
\text { stone. }\end{array}$ \\
\hline 354 & 1. 4 & .025 & .019 & & & & $\begin{array}{l}\text { Arkosic sandstone and black } \\
\text { shale. }\end{array}$ \\
\hline 355 & & .81 & .85 & & 2. 92 & 50 & $\begin{array}{l}\text { Selected high - grade ore, } \\
\text { abundant metatyuyamu- } \\
\text { nite. }\end{array}$ \\
\hline 356 & .6 & .035 & .026 & . & & & $\begin{array}{l}\text { Pink arkosic micaceous sand- } \\
\text { stone. }\end{array}$ \\
\hline 357. & 1. 6 & .010 & .003 & & & & Arkosic micaceous sandstone \\
\hline $\mathrm{BZ}-15$ & 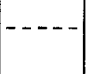 & .068 & .067 & 13. 65 & & & $\begin{array}{l}\text { Sandstone with carbonized } \\
\text { wood. }\end{array}$ \\
\hline-16 & & & & & & & Acicular limestone nodules \\
\hline $3 Z-17$ & & .004 & .0002 & 57.33 & & & Chalcocite nodules. \\
\hline BZ-18 & - & .054 & .032 & .66 & $\ldots$ & & $\begin{array}{l}\text { Sandstone with dissemin- } \\
\text { ated malachite and meta- } \\
\text { tyuyamunite. }\end{array}$ \\
\hline BZ-19 & $\ldots$ & .039 & .026 & .41 & & & $\begin{array}{l}\text { Sandstone with dissemin- } \\
\text { ated metatuyuamunite. }\end{array}$ \\
\hline
\end{tabular}

$417829-58-4$ 
TABLE 8.-Analytical data for samples from areas $G$ and $H$ (pls. 30 and 31)

[Radioactivity analyses by S. P. Furman and E. J. Fennelly. Chemical analyses by D. L. Skinner, W. N. Niles, Wayne Mountjoy, J. P. Schuch, W. D. Goss, E. C. Mallory, J. L. Siverly, C. E. Thompson, and D. L. Stockwell]

\begin{tabular}{|c|c|c|c|c|c|}
\hline \multirow{2}{*}{$\begin{array}{c}\text { Field } \\
\text { no. }\end{array}$} & \multirow{2}{*}{$\begin{array}{l}\text { Thick- } \\
\text { ness } \\
\text { (feet) }\end{array}$} & \multicolumn{3}{|c|}{ Percent } & \multirow{2}{*}{ Lithologic description } \\
\hline & & eU & U & $\mathrm{Cu}$ & \\
\hline 1 & & 0.003 & (........ & 0.63 & Gray carbonaceous shale. \\
\hline 2 & $-\ldots$ & .015 & $\ldots \ldots$ & $\ldots$ & Chalcocite nodules. \\
\hline 3 & $-\ldots$ & .005 & $1-\ldots$ & $\ldots$ & Limestone. \\
\hline 4 & $\ldots$ & .011 & 0.015 & 6. 07 & $\begin{array}{l}\text { Arkosic pebble conglomerate with chal- } \\
\text { cocite. }\end{array}$ \\
\hline 5 & $-\cdots$ & .001 & -..... & .03 & Barren micaceous sandstone. \\
\hline 6 & 6. 0 & .004 & $\ldots \ldots$ & 1. 40 & Dark-gray sandstone. \\
\hline 7 & -...- & .002 & $\ldots \ldots$ & $-\ldots$ & Barren red micaceous siltstone. \\
\hline 8 & $\mid \ldots$ & .002 & $\ldots \ldots$ & 1. 97 & Black carbonaceous shale. \\
\hline 9 & $-\ldots$ & .009 & $\ldots$ & $(-\ldots+1-10$ & Chalcocite nodules. \\
\hline 10 & $\ldots$ & .001 & - & $-\ldots$ & Barren limestone. \\
\hline 11 & $\ldots$ & .006 & .005 & 55. 73 & Wood replaced by chalcocite. \\
\hline 12 & 7. 0 & .004 & $\ldots$ & .49 & Gray silty carbonaceous sandstone. \\
\hline 14 & 2. 0 & .002 & $\ldots \ldots$ & .06 & Gray carbonaceous siltstone. \\
\hline 15 & .5 & .005 & $-\ldots$ & $\ldots \ldots$ & Do. \\
\hline 16 & $\ldots$ & .006 & $\ldots$ & $\ldots \ldots$ & Chalcocite nodules. \\
\hline 166 & 4.2 & .007 & .006 & 1. 27 & Gray and black shale. \\
\hline 167 & 2. 0 & .003 & $\ldots \ldots$ & .37 & Gray brown siltstone. \\
\hline 168 & 2. 4 & .005 & $-\ldots$ & 1. 21 & Black carbonaceous shale. \\
\hline 169 & 4. 0 & .004 & $\ldots \ldots$ & .06 & Gray limy shale. \\
\hline 170 & 2. 5 & .003 & -..... & .60 & Gray shale. \\
\hline 171 & 2.5 & .003 & $\ldots \ldots$ & 1. 49 & Black carbonaceous shale. \\
\hline 172 & 2. 0 & .002 & $\ldots \ldots$ & .05 & Gray shale. \\
\hline 174 & 3. 5 & .003 & $-\ldots$ & .03 & Gray limy shale. \\
\hline 175 & 6. 5 & .004 & $\ldots \ldots$ & .015 & Do. \\
\hline 184 & 6. 3 & .002 & $-\ldots$ & .005 & Gray and red shale. \\
\hline 185 & 3. 2 & .003 & $\ldots$ & .005 & Gray shale. \\
\hline 189 & .4 & .022 & .019 & 1. 09 & Black shale and arkose. \\
\hline 190 & 1. 5 & .005 & .001 & 3. 53 & Black carbonaceous shale. \\
\hline 191 & 3. 8 & .003 & $\ldots \ldots$ & .1 & Gray green siltstone. \\
\hline 192 & 3. 2 & .008 & .005 & 1. 05 & Gray and black shale. \\
\hline 193 & 2. 0 & .007 & .006 & .58 & Green and black shale. \\
\hline $\mathrm{BZ}-25$ & 1. 5 & .008 & .004 & 7. 49 & $\begin{array}{l}\text { Gray shaly siltstone with carbon and } \\
\text { chalcocite. }\end{array}$ \\
\hline BZ-26 & & .048 & .041 & 32.85 & $\begin{array}{l}\text { Selected radioactive chalcocite nodules } \\
\text { with uraninite. }\end{array}$ \\
\hline $\mathrm{BZ}-\mathbf{3 0}$ &.- & .015 & .008 & .80 & $\begin{array}{l}\text { Black shale with copper and limestone } \\
\text { nodules. }\end{array}$ \\
\hline
\end{tabular}


TABLE 9.-Analytical data for samples from area $I$ (pl. 32)

[Radioactivity analyses by S. P. Furman and J. H. Patton. Chemical analyses by D. L. Skinner, W. N. Niles, Wayne Mountjoy, S. P. Schuch, H. E. Bivens, W. D. Goss, R. F. Dufour, J. L. Siverly, and E. C. Mallory]

\begin{tabular}{|c|c|c|c|c|c|}
\hline \multirow{2}{*}{$\begin{array}{c}\text { Field } \\
\text { no. }\end{array}$} & \multirow{2}{*}{$\begin{array}{l}\text { Width } \\
\text { (feet) }\end{array}$} & \multicolumn{3}{|c|}{ Percent } & \multirow{2}{*}{ Lithologic description } \\
\hline & & $\mathrm{eU}$ & U & $\mathrm{Cu}$ & \\
\hline 122 & 2. 5 & 0.004 & . & 0.14 & Gray-green carbonaceous shale. \\
\hline 123 & 3. 5 & .004 &.- & .32 & Do. \\
\hline 124 & 1. 7 & .003 & -1 & .05 & Olive-brown micaceous shale. \\
\hline 125 & 2. 0 & .004 & $\ldots \ldots$ & .02 & Gray-brown and gray-green shale. \\
\hline 126 & 1.3 & .005 & - & .03 & Light-gray thin-bedded arkose. \\
\hline 127 & 2. 0 & .009 & 0.006 & .75 & Gray-green micaceous siltstone. \\
\hline 128 & 3. 0 & .003 & $\ldots$ & .02 & Gray-green shale. \\
\hline 129 & 2. 0 & .003 & $\ldots \ldots$ & .02 & Gray-green micaceous siltstone. \\
\hline 130 & 1. 2 & .004 & $\ldots \ldots$ & .01 & Do. \\
\hline 131 & 1. 3 & .005 & - & .01 & Olive clay shale and siltstone. \\
\hline 132 & 2. 0 & .005 & & $<.01$ & Olive-brown micaceous siltstone. \\
\hline 133 & 2. 7 & .002 & 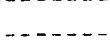 & $<.01$ & Reddish arkosic pebble conglomerate. \\
\hline 134 & 2. 0 & .004 & $\ldots \ldots$ & .01 & Gray-green micaceous siltstone. \\
\hline 135 & 3. 4 & .004 & $\ldots$ & .65 & Do. \\
\hline 136 & 1. 5 & .012 & .008 & 2. 10 & Gray-green siltstone. \\
\hline 137 & 2. 7 & .005 & $\ldots \ldots$ & .81 & Black carbonaceous shale. \\
\hline 138 & 2. 0 & .004 & 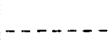 & 1. 52 & Green micaceous siltstone. \\
\hline 139 & 1. 0 & .003 & $\ldots$ & 1. 47 & Green siltstone. \\
\hline 141 & $-\ldots$ & $\ldots$ & $\ldots$ & $\ldots$ & Chalcocite nodules. \\
\hline 142 & 1. 9 & .004 & & 1. 79 & Gray-green siltstone. \\
\hline 143 & 4. 8 & .003 & $-\ldots$ & .01 & Gray-green clay shale. \\
\hline 144 & 2. 0 & .004 & - & .07 & Gray-green micaceous siltstone. \\
\hline 145 &.- & $\ldots$ & $\ldots$ & $\ldots$ & Linoproducts sp. (brachiopod). \\
\hline 146 & 5. 0 & .003 & 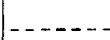 & .01 & Gray-green shale. \\
\hline 147 & $\cdots$ & $\ldots$ & & $\ldots$ & Poorly sorted arkose with malachite. \\
\hline 148 & $\ldots$ & & - & 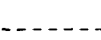 & Coaly, iron-stained log with copper. \\
\hline 149 & $\ldots$ & $\ldots$ & & $\ldots$ & Log, partly replaced by chalcocite. \\
\hline 150 & .8 & .004 & - & .54 & Gray-green clay shale. \\
\hline 151 & .6 & .002 & $\ldots \ldots$ & .66 & Green and brown clay shale. \\
\hline 152 & 4. 2 & .005 & $\ldots \ldots$ & 1. 57 & Carbonaceous shale and siltstone. \\
\hline 153 & 3. 5 & .008 & .005 & 1. 74 & Do. \\
\hline 154 & 2. 4 & .008 & .005 & 1. 46 & Carbonaceous sandstone and siltstone. \\
\hline 155 & 2. 0 & .007 & .005 & 1. 85 & Carbonaceous shale. \\
\hline 156 & 1. 6 & .005 & 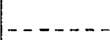 & 5. 20 & Gray-green carbonaceous shale. \\
\hline 157 & 1. 0 & .003 & & .02 & Dark-gray and brown shale. \\
\hline 158 & 2. 7 & .005 & $5-$ & 2. 04 & Gray-green carbonaceous shale. \\
\hline 159 & 1. 1 & .007 & - & 1. 55 & Gray-green clay shale. \\
\hline 160 & 2.2 & .003 & & 1. 44 & Gray shale and arkose. \\
\hline 161 & 3. 0 & .004 & - & .05 & Gray shale with red mottling. \\
\hline 162 & 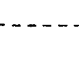 & $\ldots$ & & 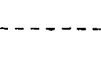 & $\begin{array}{l}\text { Hypselentoma and bellerophontid gas- } \\
\text { tropods. }\end{array}$ \\
\hline BZ-28 & .3 & .054 & .061 & 3. 84 & Gray shaly sandstone. \\
\hline BZ-29 & .5 & .011 & .003 & 2. 23 & Carbonaceous siltstone and shale. \\
\hline
\end{tabular}


TABLE 10.-Analytical data for samples outside areas of large-scale maps

[Samples listed in order of appearance on map (pl. 24) from south to north. Radinactirity analyses by S.P. Furman and E.J. Fennelly. Chemical analyses by D. L. Skinner, J. H. MeCarthy, W. N. Niles, Wayne Mountjoy, J. P. Schuch, and W. D. Goss]

\begin{tabular}{|c|c|c|c|c|c|}
\hline \multirow{2}{*}{$\begin{array}{c}\text { Field } \\
\text { no. }\end{array}$} & \multirow{2}{*}{$\begin{array}{l}\text { Width } \\
\text { (feet) }\end{array}$} & \multicolumn{3}{|c|}{ Percent } & \multirow{2}{*}{ Lithologic description } \\
\hline & & $\mathrm{eU}$ & U & $\mathrm{Cu}$ & \\
\hline$B Z-31$ & -- & 0.003 & 0. 002 & 5. 24 & Sandstone with carbon. \\
\hline$B Z-32$ & & .004 & .003 & 6. 76 & Dark-gray carbonaceous clay shale. \\
\hline 8 & ... & .002 & .... & 1. 97 & Carbonaceous shale. \\
\hline 163 & 1. 5 & .002 & $\ldots \ldots$ & .01 & Gray shale. \\
\hline 164 & 1. 0 & .003 & $-\ldots-$ & .19 & Light and dark-gray shale. \\
\hline 165 & 1. 4 & .003 & $\ldots$ & .06 & Gray shale with carbon. \\
\hline$B Z-27$ & $\ldots$ & .003 & .002 & .02 & Greenish-gray clay shale. \\
\hline$B Z-24$ & 2. 0 & .003 & $<.001$ & .005 & Barren greenish-gray clay shale. \\
\hline 20 & - _- & $\ldots$ & $\ldots \ldots$ & $\ldots$ & Unaltered chalcocite nodules. \\
\hline $21^{1}$ & $\ldots$ & .003 & $\ldots$ & 1. 06 & Fossiliferous limestone. \\
\hline$B Z-22$ & & - & & $\ldots$ & Marine fossils in sandy limestone. \\
\hline$B Z-23$ & & $\ldots-$ & & - n & Do. \\
\hline$B Z-21$ & $\cdots$ & .005 & .003 & .005 & Greenish-gray clay shale. \\
\hline 25 & - - & .001 & $-\ldots$ & $-\ldots$ & Gray-green and red shale. \\
\hline$B Z-20$ & 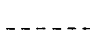 & .009 & .004 & 1. 81 & Carbonaceous shaly sandstone. \\
\hline $26^{1}$ & 1. 2 & .003 & $\ldots$ & 2. 00 & Gray-green carbonaceous shale. \\
\hline 27 & 1.2 & .004 & & & Do. \\
\hline 28 & $\cdots$ & .001 & $\ldots$ & 3. 46 & Light arkosic sandstone. \\
\hline 29 & & $\ldots$ & & $\ldots \ldots$ & Mineralized carbonaceous wood. \\
\hline 31 & $\ldots$ & .003 & - - - - & $\ldots$ & Silicified wood with copper. \\
\hline 32 & 3. 0 & .003 & $\ldots$ & 1. 27 & Gray carbonaceous shale. \\
\hline 53 & 5. 7 & .003 & . & .08 & Red siltstone, gray micaceous shale. \\
\hline 54 & $-\ldots$ & .004 & 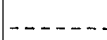 & 0.84 & Gray-green siltstone and shale. \\
\hline 52 & $\ldots \ldots$ & .005 & & - $\ldots \ldots$ & Brown fine-grained sandstone. \\
\hline $49^{1}$ & 3. 0 & .003 & & .02 & Green shale with copper. \\
\hline $50^{1}$ & 8. 7 & .003 & $\ldots$ & .76 & Do. \\
\hline 51 & $\ldots$ & .003 & - & $\ldots \ldots$ & Gray-green shale with copper. \\
\hline$B Z-6$ & $\ldots$ & .002 & .001 & .005 & Greenish-gray clay shale. \\
\hline$B Z-5$ & $\ldots$ & .018 & .014 & 6. 13 & Black carbonaceous shale. \\
\hline 55 & & .009 & & $\ldots$ & Carbonaceous shale. \\
\hline 56 & - & .005 & - & - n & Gray-green shale from dump. \\
\hline 57 & & $\ldots$ & & $\ldots \ldots$ & Chalcocite nodules and replaced wood. \\
\hline
\end{tabular}

1 Semiquantitative spectrographic analyses given in table 11.

The copper deposits are confined to sedimentary rocks that probably were deposited in small, stagnant basins. The association of the copper deposits and this sedimentary facies is so distinct that a genetic relationship is indicated. Most of the copper deposits are in black shale lenses in the transition unit. The black shale lenses are enclosed in gray-green shale that contains a small amount of copper and grades into barren red shale or limestone. Detailed sections of two typical ore bodies are shown in figures 71 and 72 . The sequence of rock types shown in figure 71 probably marks successive stages 
COPPER AND URANIUM DEPOSITS, COYOTE DISTRICT, N. MEX. 367

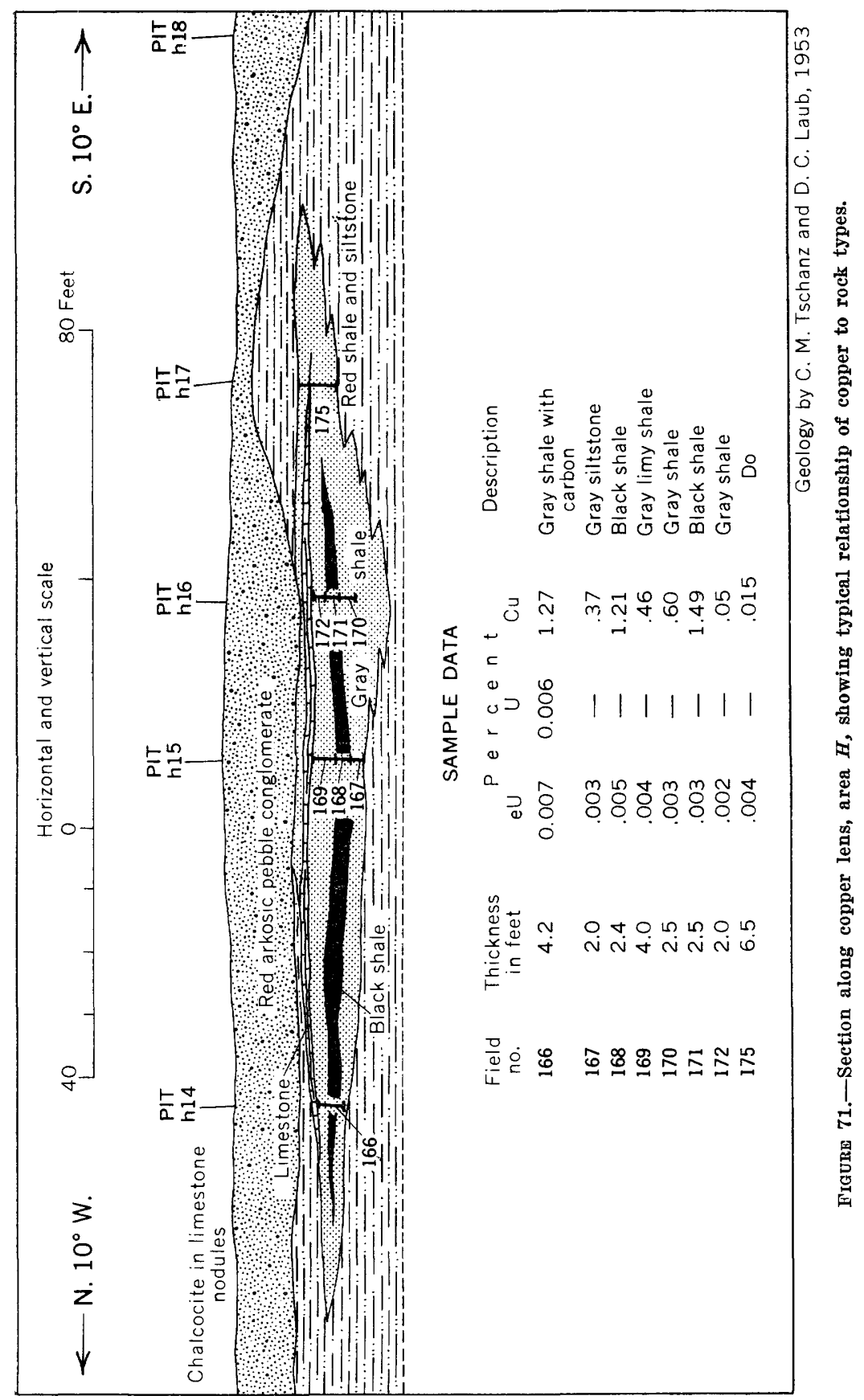




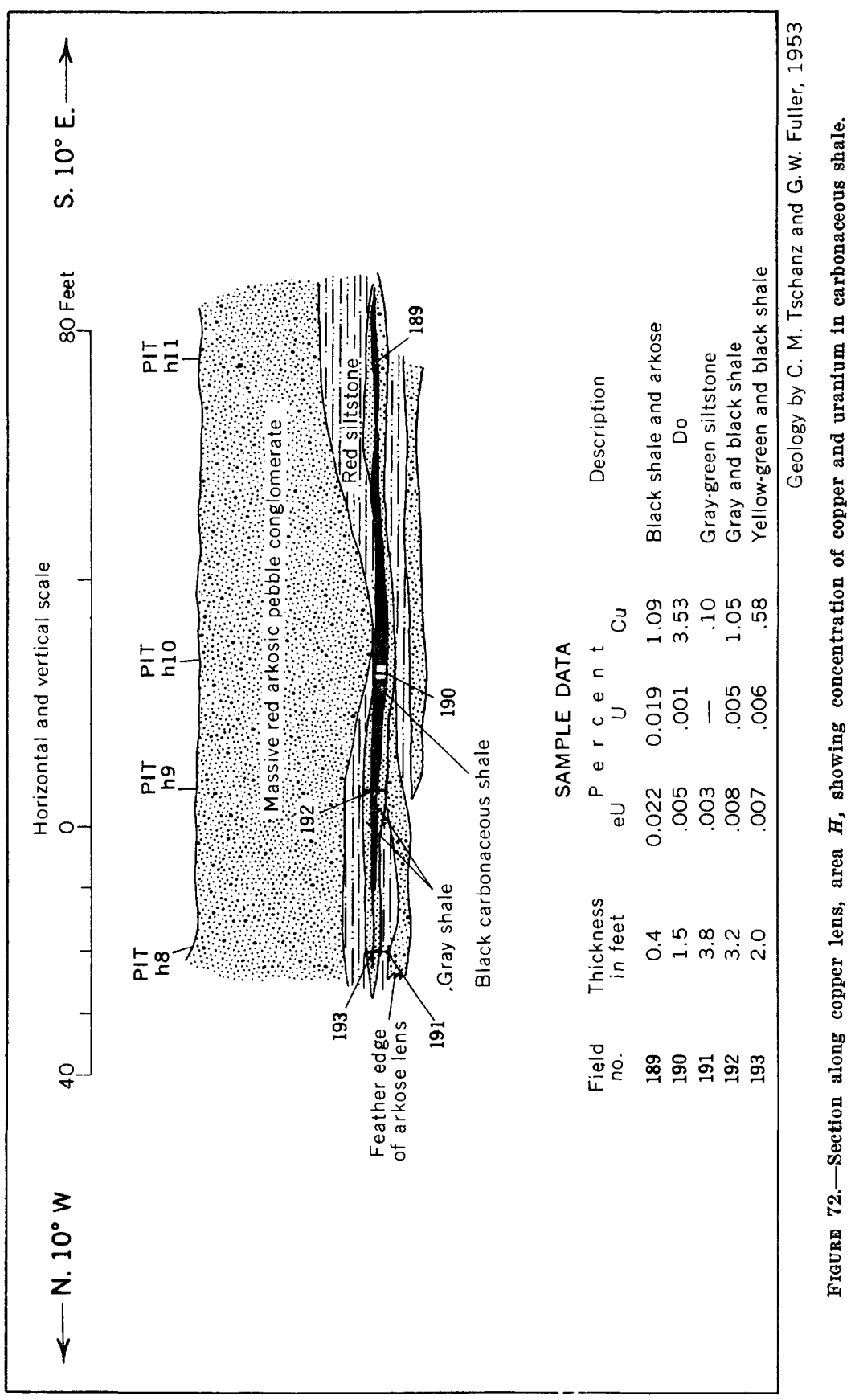


in the filling of a local basin. The limited extent of the favorable host rock, the black shale, limits the size of the ore bodies.

The ore bodies are tabular bedded deposits 100-300 feet in diameter and 1 to 6 feet thick. The copper content ranges from 1 to 6 percent. The average ore lens is 2 or 3 feet thick and contains about 1.5 percent copper. None of the deposits contains more than about 7,500 tons of ore, and few contain more than 1,000 tons.

The ore contains chalcocite nodules less than 2 inches in diameter, but some as large as a man's head have been reported. Most nodules are not radioactive, although a few highly radioactive ones were found at pits G2, E15, and E16.

\section{MINERALOGY}

The principal copper minerals are blue and gray chalcocite, malachite, and azurite. Azurite and malachite commonly occur as disseminated grains or coatings on chalcocite nodules or mica flakes. The chalcocite nodules contain small amounts of pyrite, bornite, covellite, and vitrain. Uraninite was identified in a chalcocite nodule from pit G2, plate 30, by Abraham Rosenzweig of the Atomic Energy Commission (Zeller and Baltz, 1954, p. 5). Volborthite is present with malachite in a micaceous arkosic sandstone in pit e5, plate 28.

A woody cell structure and even the form of the woody fragments are well preserved in many chalcocite nodules (pl. 33), and all stages of replacement by chalcocite can be found. Normally the cell walls are vitrain and the cores are filled with sulfide minerals. A few nodules have a concentric banded structure that may be marked by slender groups of pyrite crystals radiating from the center.

Chalcocite also occurs in dense limestone nodules near pits b14 and a62. In a few places the dense limestone core of the nodules is surrounded by concentric layers of coarse, acicular brownish calcite that contains malachite. Chalcocite is rare in sandstone but the outer edge of a pebble conglomerate bed adjacent to copper-bearing shale may contain as much as 14 percent copper, chiefly chalcocite.

Many coalified woody fragments were composed entirely of vitrain and pyrite before pyrite was replaced by copper sulfides. Bornite and chalcopyrite are most abundant in nodules that still contain abundant pyrite. Covellite and chalcocite are the important minerals where replacement is nearly complete ( $\mathrm{pl}$. 33). The paragenetic sequence is: (1) vitrain and pyrite, (2) chalcopyrite and bornite, (3) chalcocite, and (4) covellite. Malachite and azurite are believed to be products of weathering. The paragenetic sequence is in the order of increasing copper content and decreasing iron content of the sulfide minerals. The chalcocite (possibly digenite(?)) is distinctly blue in some nodules where it has almost completely replaced pyrite, bornite, and chalcopyrite (pl. 33). The blue color is typical 
of a chalcocite-covellite solid solution, which may contain as much as 8 percent covellite at room temperature. The blue chalcocite is interpreted by some to indicate a temperature of formation above $75^{\circ} \mathrm{C}$ (Edwards, 1947 , p. 74-76). Copper-bearing samples contain less vanadium, lead, and rare earths than uranium samples (table 11, page 372$)$.

\section{URANIUM DEPOSITS}

\section{URANIUM DEPOSITS IN SANDSTONE}

The uraniferous shale and siltstone are of very low grade and all commercial-grade uranium deposits are in sandstone. The uranium deposits may or may not contain significant amounts of copper or vanadium.

Uranium and vanadium minerals are commonly concentrated in scours filled by sandstone that contains abundant clay and rock fragments and carbonized wood (fig. 73). Most of the ore pockets are less than 2 feet thick and 10 feet long. A group of several closely spaced deposits within a favorable sandstone generally are separated from other groups by long stretches of barren sandstone. The ore pockets are surrounded by lower grade material, and radioactivity may be continuous between adjacent deposits. The outcrops of the ore bodies are inconspicuous. Metatyuyamunite is locally abundant, but recognizable uranium minerals are not visible in many deposits.

The uranium deposits in sandstone are described below.

Area $B$, Blas Medina property.-All the uranium deposits in area $B$ are probably in the same gently dipping arkosic sandstone about 1,670 feet above the base of the formation. Uranium was found in all the

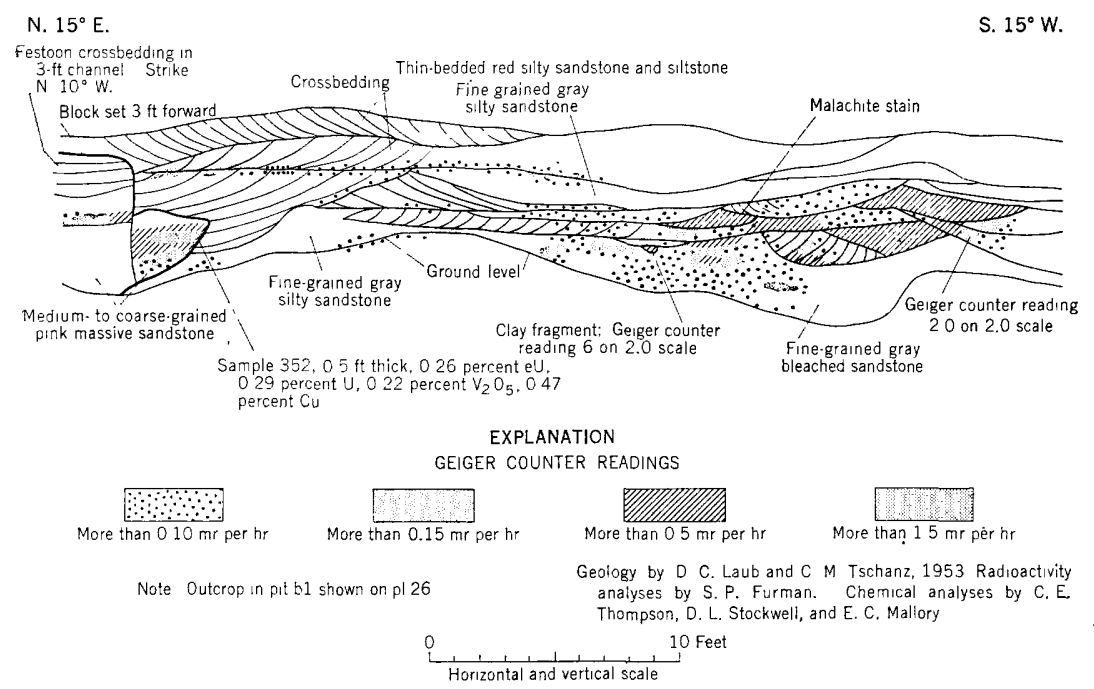

FIGURE 73.-Section along outcrop of ore sandstone, Blas Medina property, Coyote district, Mora County, N. Mex. 
trenches except b5 and b7, but ore was found only in trenches b1, b4, and the new shaft. The map of these deposits is given in plate 26 and the sample analyses are given in table 4 .

The ore in pit b4 is in a hard, limy, massive, medium-grained, pink. sandstone which fills a scour about 10 feet in diameter. The ore, 4 to 6 . inches thick, contains abundant small grains of disseminated chalcopyrite; metatyuyamunite is rare. Chalcopyrite has been found only in area $B$.

In the summer of 1955 a 15 -foot shaft was sunk by lessees about 25 . feet behind the outcrop at pit $b 4$. They reported that the lowest part of the shaft cut through several feet of ore containing abundant chalcopyrite and as much as 1 percent equivalent uranium. A grab sample (360) analyzed in the laboratories of the Geological Survey contained 0.78 percent equivalent uranium and 0.41 percent uranium (table 11), and duplicate analyses confirmed these figures. The difference between the radiometric and chemical values was surprisingly great compared to sample 251 from the nearby outcrop which was: nearly in equilibrium. Radiochemical analyses for uranium daughter elements were made by J. N. Rosholt, Jr., to determine the cause of the disequilibrium. The radiochemical data (table 11) are interpreted to mean that uranium probably has been selectively leached from sample 360 even though the sample contains abundant chalcopyrite. This conclusion is surprising because uranium has apparently not been comparably leached from the surface outcrop (251). If this interpretation is accepted, the ore must have originally contained more uranium, perhaps as much as the 1.34 percent required to form the ionium in the sample. The indicated subsurface leaching of uranium suggests that uranium is readily soluble under certain conditions where chalcopyrite is relatively stable. The uranium leached from the ore might possibly be redeposited in minable concentrations downdip. The only alternative interpretation permitted by the radiochemical data would have ionium extracted by the sample from ground waters by some mechanism such as coprecipitation with or adsorption on ferric hydroxides. This alternative is unlikely.

In pit b1, figure 73, several sets of cross strata in the sandstone are separated by curved, troughlike erosion surfaces. Uranium is concentrated along clay seams, in clay fragments, or along discontinuities between cross strata. In the spring of 1954, radioactive material was discovered in two massive, grayish-pink arkosic sandstone beds from 1,000 to 1,300 feet north of pit b1. The sandstone beds, $6-8$ feet thick, are separated by 20 feet of siltstone. Fine-grained malachite, the only visible ore mineral, is probably derived from chalcopyrite. The radioactive layer in the center of each sandstone bed is $1-2$ feet thick. Readings as much as 0.2 milliroentgens per hour on the Geiger counter were obtained, but samples were not collected. 


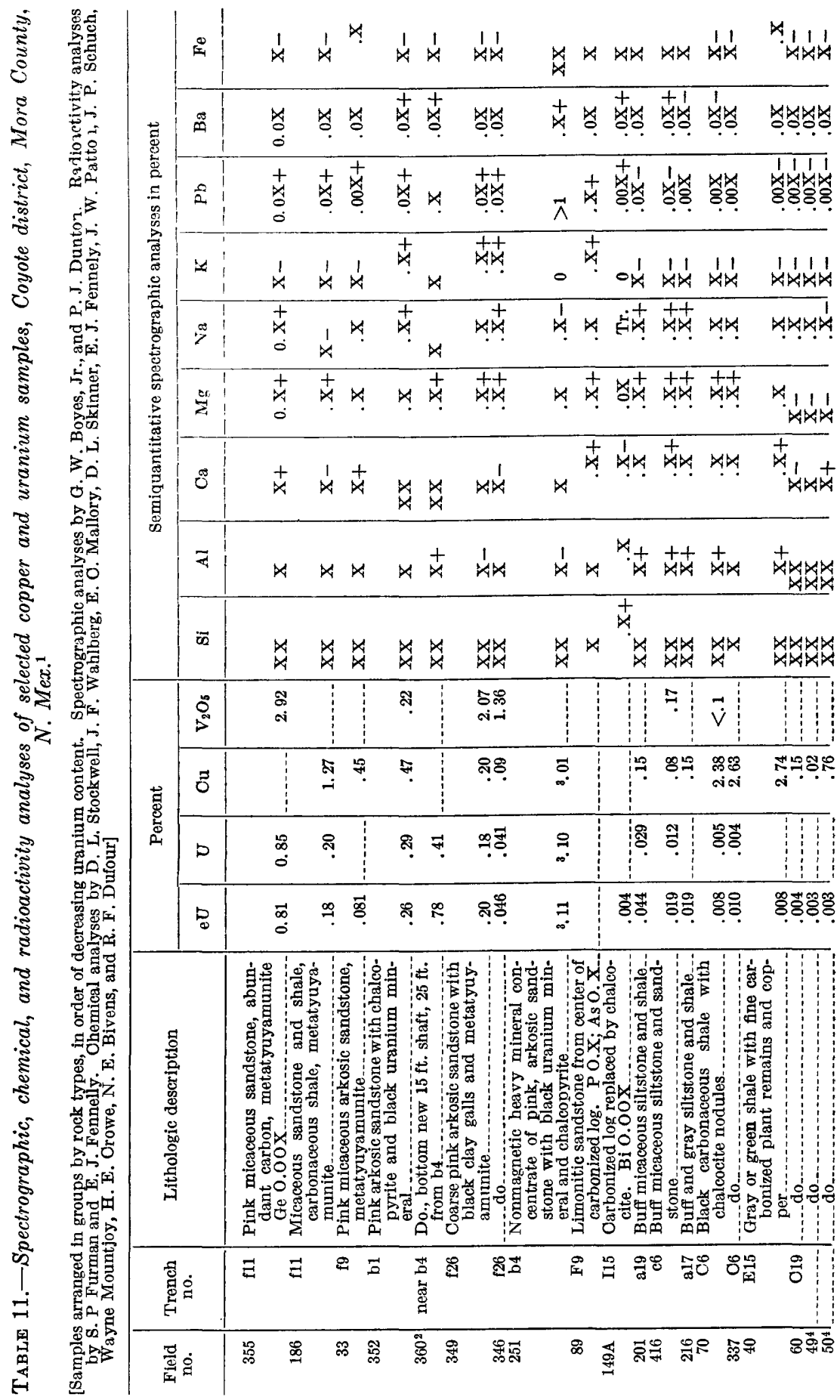


COPPER AND URANIUM DEPOSITS, COYOTE DISTRICT, N. MEX. 373

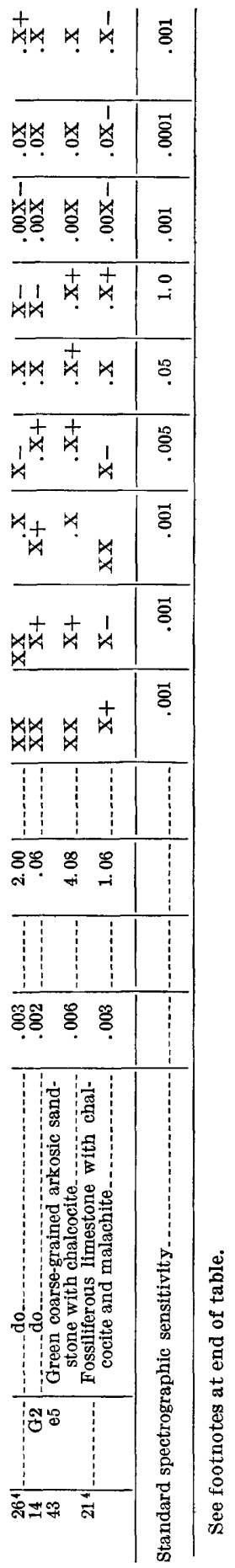


374 CONTRIBUTIONS TO THE GEOLOGY OF URANIUM

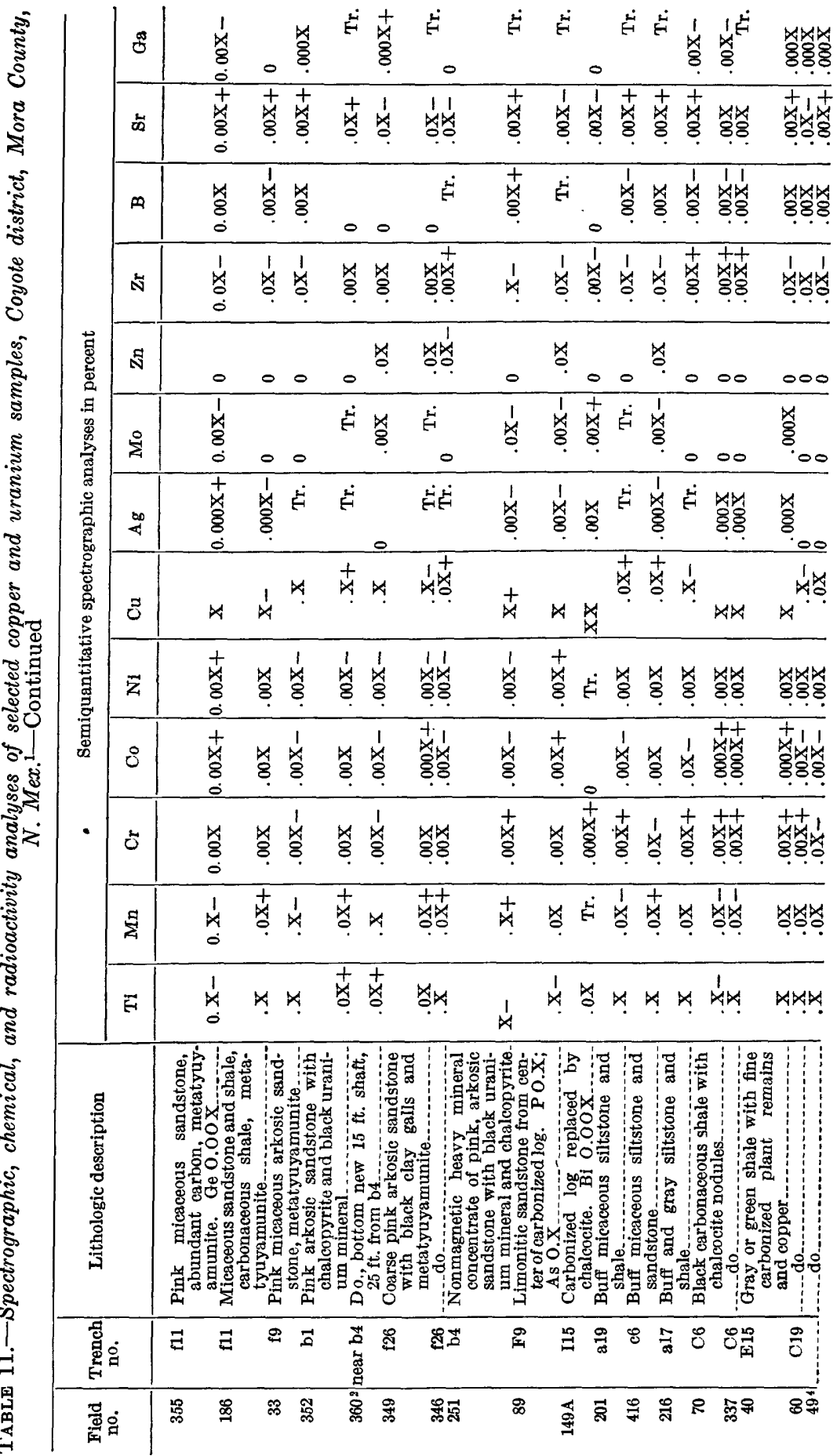


COPPER AND URANIUM DEPOSITS, COYOTE DISTRICT, N. MEX. 375

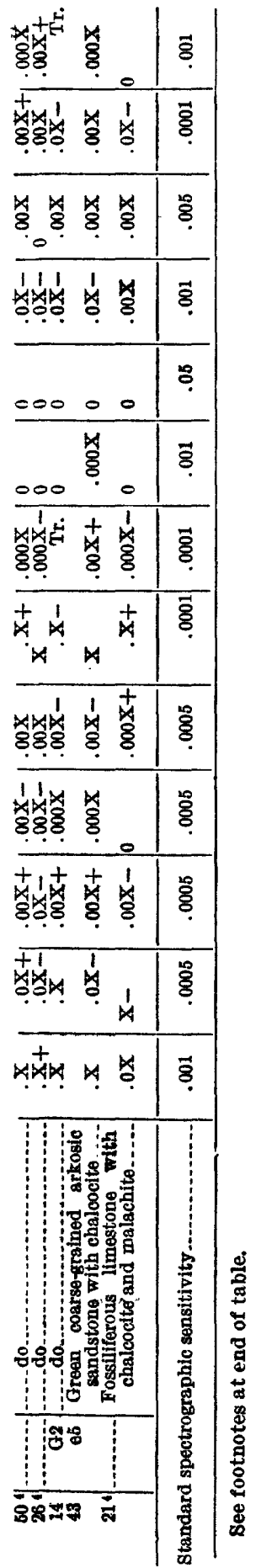




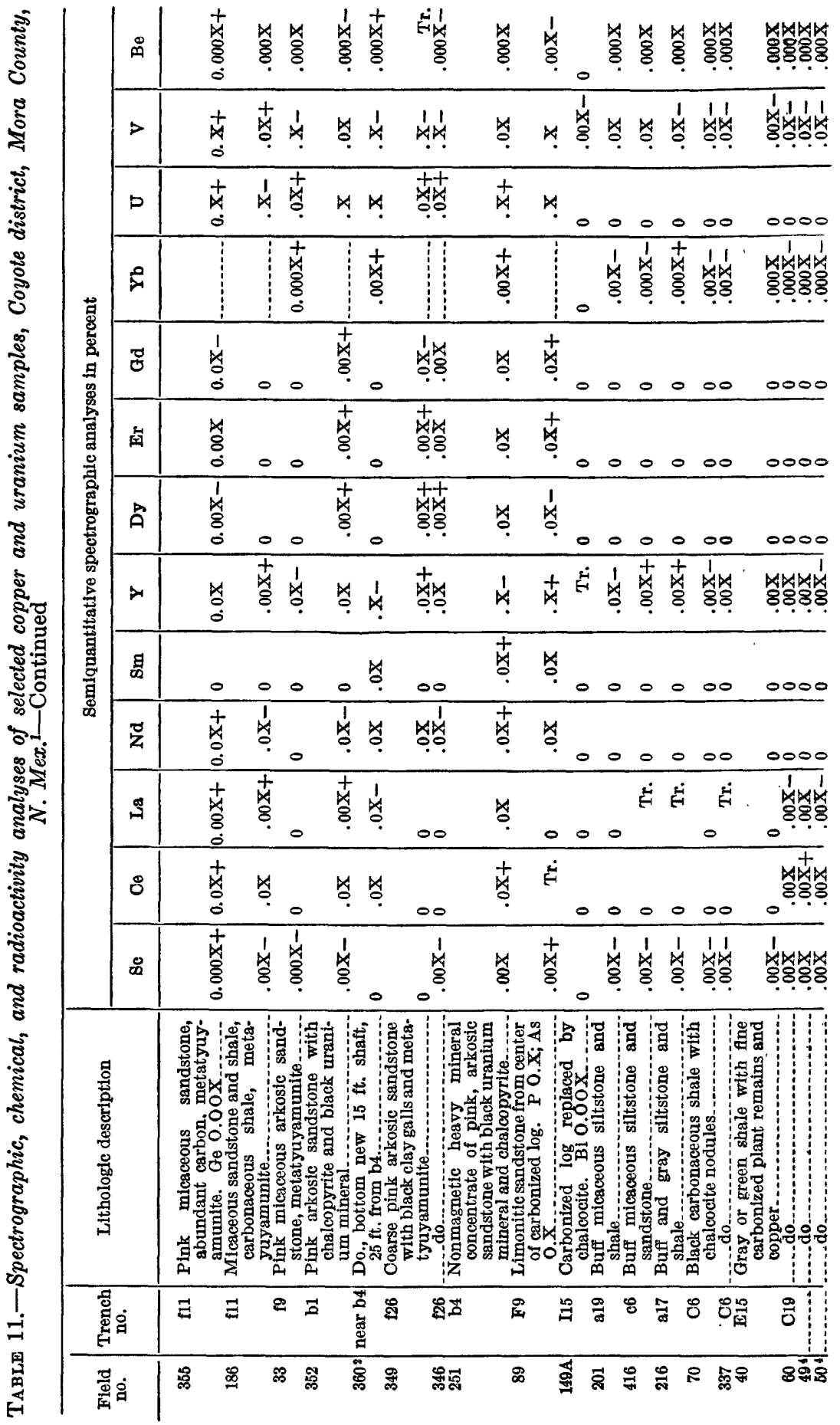


COPPER AND URANIUM DEPOSITS, COYOTE DISTRICT, N. MEX. 377

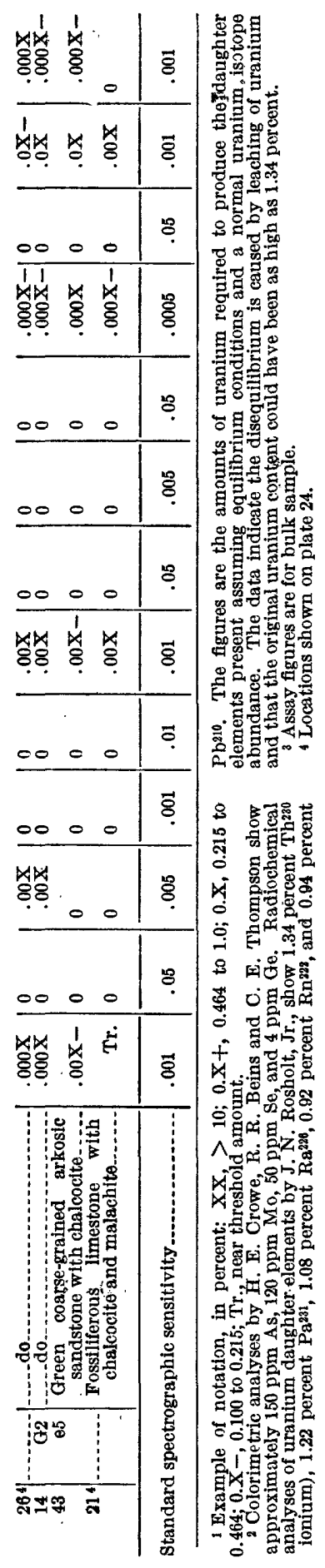


Area $F$, Arturo Le Deux property.-Massive pink medium-grained limy micaceous arkosic sandstone contains uranium and vanadium between pits $\mathrm{f} 9$ and $\mathrm{f} 12$ (pl. 29). The sandstone ranges in thickness from a featheredge to 4 feet and crops out sporadically at the surface. This sandstone is about 770 feet above the base of the formation and is enclosed in copper-bearing gray shale containing thin seams of black shale. Metatyuyamunite is concentrated in micaceous seams at the contact of the sandstone and the shale in pit f11 and disseminated through the sandstone in pit f9. A high-grade ore pocket found 2 feet below the surface in the featheredge of the sandstone in pit f11 probably extends to the north end of pit 12 . The ore between depths of 2 and 4 feet is less than 2 feet thick. The radioactivity is not above background count south of pit $\mathrm{f} 12$ and north of pit $\mathrm{f} 8$, and no other uranium deposits were found in this zone. The analyses in table 7 indicate that uranium has been leached from the uraniferous shale and redeposited in the adjacent sandstone. The lack of control by sedimentary structures within the sandstone and the close association with copper-bearing shale are exceptional.

Three nearly vertical fluviatile sandstone beds between 1,920 and 1,960 feet above the base of the Sangre de Cristo formation contain uranium or vanadium deposits in the eastern part of area $F$. The middle or main uranium-bearing sandstone is a gray-brown poorly sorted, massive limy sandstone about 6 or 8 feet thick. This sandstone contains isolated concentrations of uranium in each pit between $f 19$ and f26. Pockets of uranium ore occur chiefly in poorly defined lenses of coarse, pink, limy sandstone that contains carbonized wood and gray or black clay and limestone fragments. The pink color is distinctive and becomes more intense as the radioactivity increases. Black micaceous vanadium minerals impregnate the sandstone along bedding planes and partly surround the uranium pockets. The black color of clay fragments in ore of high vanadium content probably is the result of adsorbed vanadium, but at least part of the vanadium is in the clay mineral structure. The ore-grade material is less than a foot thick except in pits f23 and $\mathbf{f 2 6}$ where metatyuyamunite is abundant.

Several vertical slickensided joints cut the sandstone between pits f23 and f26. One joint contains nonradioactive caliche and iron oxides. A narrow zone of sandstone adjacent to this joint is barren; beyond this a zone impregnated with black vanadium minerals is parallel to the joint and partly envelops a small uranium pocket.

The lowest of the three sandstone beds contains small vanadium deposits, but the uranium content is low. The highest of the three sandstone beds is represented by a group of small isolated hard white massive fine-grained limy sandstone lenses at about the same stratigraphic position above the main ore-bearing sandstone. Several of 


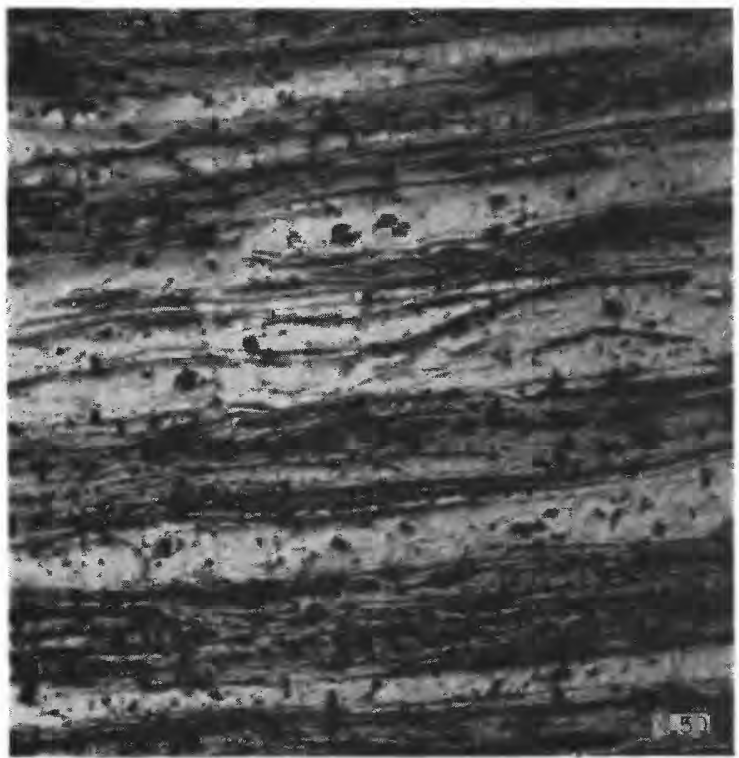

A. Polished longitudinal section of carbonized wood (dark gray) replaced by blue chalcocite (gray) containing residual grains of pyrite and bornite (not visible). X 50 .

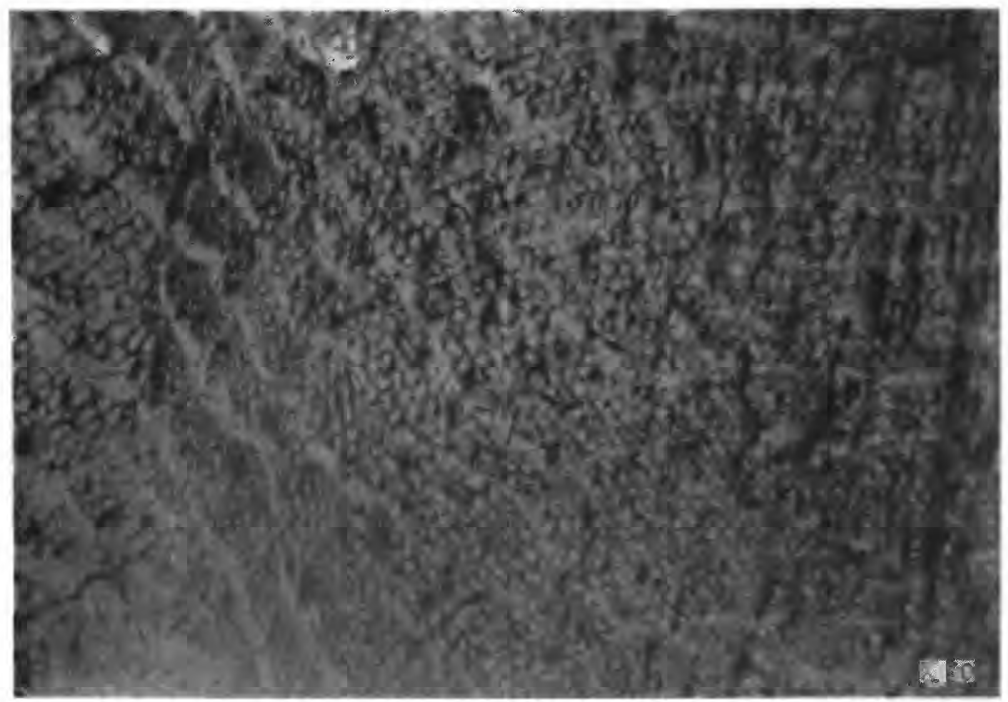

B. Polished cross section of carbonized wood (dark gray) replaced by gray chalcocite (gray) and minor covellite (not visible). Shows cell structure and growth rings. X 50 .

PHOTOMICROGRAPHS OF COPPER ORES, COYOTE DISTRICT, MORA COUNTY, N. MEX. 


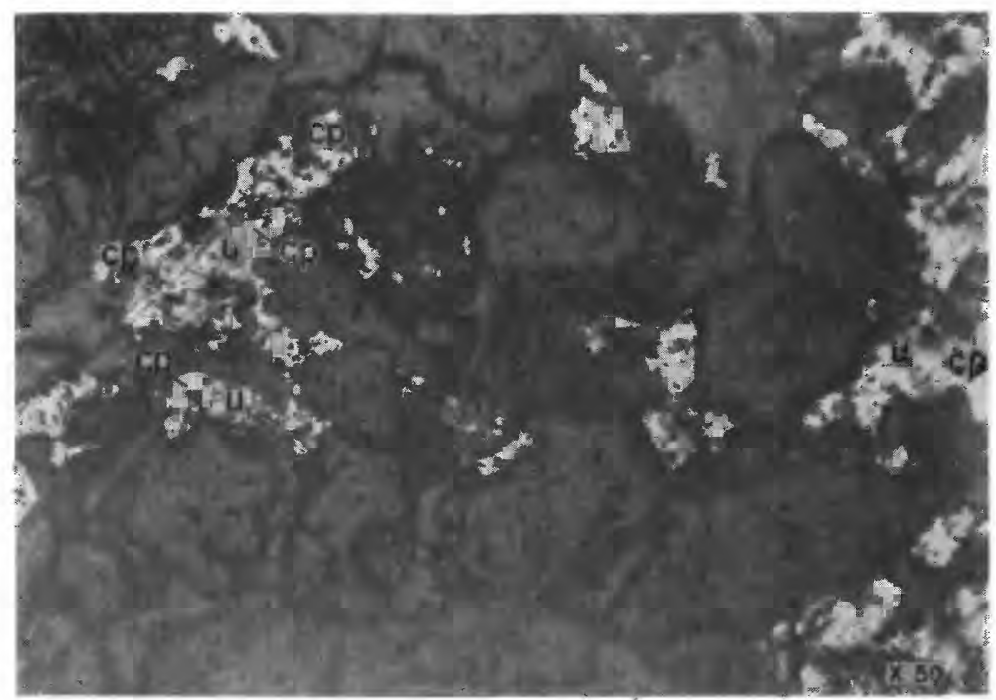

A. Polished section of uraniferous sandstone, Blas Medina property, pit b-4. Quartz and feldspar (high relief) and calcite (low relief) replaced by chalcopyrite (cp) and black uraniferous substance $(u)$. X 50 .

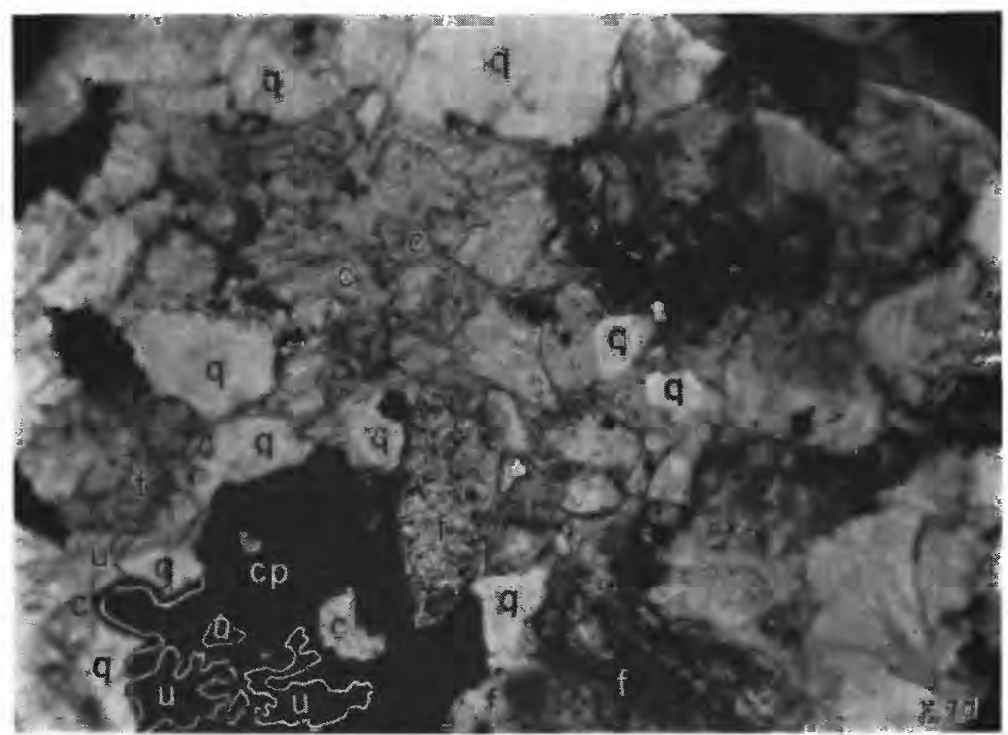

$B$. Thin section of hematitic uraniferous sandstone, Blas Medina property, pit b-4. Quartz ( $q$ ), hematite-impregnated feldspar $(f)$, and calcite (c), replaced by chalcopyrite and black uraniferous substance. $\quad$ X 70 .

PHOTOMICROGRAPHS OF URANIFEROUS SANDSTONE, COYOTE DISTRICT, MORA COUNTY, N. MEX. 
these lenses contain uranium but no copper or vanadium. The part of the lenses that contains uranium is pink, as in pit $\mathrm{f} 28$, $\mathrm{f} 32$, and $\mathrm{f} 33$.

An ore-grade layer 3 to 5 inches thick parallels a slickensided bedding plane in the middle of a 3 -foot massive limy sandstone bed in pits $\mathrm{f} 32$ and f33. The layer is apparently continuous between these pits and may extend 50 feet farther north. The deep pink ore-grade material grades into barren white sandstone.

\section{URANIFEROUS SILTSTONE AND SHAIE}

A uraniferous micaceous marine siltstone and shale bed overlain by a thin bed of fossiliferous marine limestone in area $A$ is correlated with a similar uraniferous rock sequence in area $C$. If this correlation is correct, the basal 330 feet of the Sangre de Cristo formation is missing in area $C$ because 670 feet of beds intervene between the uraniferous siltstone and the apparent base of the formation in area $A$, but only 340 feet intervene in area $C$. If the uraniferous siltstone beds are correlated, the uraniferous sandstone beds in area $F$ can be correlated with those in area $C$. The discrepancy in thickness of the basal beds may be due to nondeposition or, more probably, faulting that has cut out the basal part of the formation in area $C$.

The uraniferous siltstone averages 2 feet in thickness and can be traced more than 5,000 feet in area $A$ and 1,500 feet in area $B$. The bed is exposed downdip for 200 feet. Samples from many trenches show that the lithologic character and the uranium content are remarkably uniform (see table 3). Nine segments of this bed in area $A$ (pl. 25) and one in area $C$ (pl. 27) contain an average of 0.01 percent uranium. Elsewhere the uranium content is about half as much.

The uraniferous beds cannot be traced between areas $A$ and $C$. Near the south end of area $A$ the siltstone grades into radioactive sandstone that contains some malachite. In places the siltstone facies between areas $A$ and $C$ is replaced by sandstone.

\section{MINERALOGT OF URANIUM DEPOSTTS}

The chief ore minerals in the uranium deposits in sandstone are metatyuyamunite, an unidentified uraniferous substance, black micaceous vanadium minerals similar to "roscoelite," chalcopyrite, and malachite. Uraninite has not been identified in any of the uranium deposits in sandstone. No other uranium and vanadium minerals have been recognized in the uraniferous siltstone.

Most of the uranium in area $B$ is apparently in hematite(?) and especially in a black uraniferous ferric(?) substance that gives positive tests for uranium, iron, and copper but does not give an X-ray diffraction pattern. Not all the black "mineral" contains uranium but it is difficult to distinguish between the two varieties visually. In oblique light fragments or irregular surfaces of the uraniferous substance appear black, but polished surfaces in a light source perpendicu- 
lar to the surface appear gray. Grains viewed under either oblique or vertical light commonly show a red internal reflection. Under the microscope the uraniferous substance is seen to be interlocked with chalcopyrite, which it apparently replaces (pl. 34). Both minerals replace calcite and, to a lesser extent, quartz and altered feldspar. Both minerals occur chiefly as finely disseminated grains that clearly were formed later than calcite, which was formed later than the sand grains. A few detrital grains contain chalcopyrite and a black substance, but the amount of metal so deposited is negligible.

A heavy-mineral concentrate, sample 251, is composed of chalcopyrite (50 percent), the unidentified substance ( 30 percent), and small amounts of quartz, feldspar, tourmaline, and accessory minerals. This sample was submitted for semiquantitative spectrographic analyses after crusher steel was removed (table 11). The unidentified uraniferous substance is probably a metamict or amorphous ferric oxide. Calculations from semiquantitative spectrographic data indicate that the uranium content of the uraniferous ferric oxide is less than 3 percent. It is suggested that the difference in physical properties between the black uraniferous ferric substance and the hematite may be the result of changes caused by radiation from a much greater uranium content.

Most of the sedimentary rocks of the Sangre de Cristo formation are feldspathic. The composition, in percent, of the ore-bearing sandstone is quartz $(30-40)$, feldspar $(30-40)$, calcite $(20-30)$, muscovite $(0-5)$, rock fragments $(0-20)$, and a little hematite and nontronite. The feldspar is chiefly oligoclase and microcline. Although potash feldspar was not detected in the five samples studied by X-ray diffraction techniques, much of the feldspar shows the characteristic gridiron pattern of microcline, and orthoclase may be present.

Except in the upper uraniferous sandstone in area $F$, the calcite content of the radioactive sandstone generally is higher than the content of nonradioactive sandstone. Calcite replaces altered feldspar and quartz; it also fills fractures in these minerals. Where replacement is advanced, the detrital grains are represented by corroded remnants that are no longer in contact. Some of the calcite in area $F$ is high in manganese. The genetic significance of these facts is discussed on page 388.

The pink color of some uraniferous sandstone ore is caused by "dusty" grains of hematite (?) which coat quartz grains and impregnate altered feldspar grains and foreign rock fragments. Most of the "dusty" hematite( ?) tested gives a positive test for uranium.

\section{CHEMICAI CHARACTERISTICS OF URANIUM-BEARING SANDSTONE}

The amount and relative proportions of uranium, vanadium, copper, and certain minor constituents vary greatly, as shown by the selected analytical data in tables 11 and 12. 
In general, the uraniferous sandstone of the Coyote district has a higher content of copper, lead, rare earths, and other minor constituents than the uranium ores in the Morrison and Shinarump formations of the Colorado Plateau. Lead and the rare earths are concentrated with uranium in the radioactive sandstone and in the heavymineral fraction (sample 251, table 11). The highest rare-earth content is found in the limonitic sandstone core of a carbonized log, sample 89. The rare-earth content of this sample exceeds the uranium and vanadium content. The few selenium analyses (tables 7 and 12) suggest that the selenium content is higher in the uraniferous rocks, particularly in the most uraniferous sandstone, but the data are not conclusive.

\section{TABLE 12.-Chemical characteristics of uranium-bearing sandstone}

[Radiozotivity analyses by S. P. Furman, chemical analyses by C. E. Thompson, D. L. Stockwell, E. C. Mallory, J. F. Wahlberg, Wayne Mountjoy, J. P. Schuch, W. D. Goss, J. H. McCarthy, H. E. Bivens, R. F. Dufour, and J. L. Siveriy]

\begin{tabular}{|c|c|c|c|c|c|c|c|c|c|}
\hline \multirow{2}{*}{$\begin{array}{c}\text { Field } \\
\text { no. }\end{array}$} & \multirow{2}{*}{$\begin{array}{l}\text { P1t } \\
\text { no. }\end{array}$} & \multirow{2}{*}{ Lithologic description } & \multicolumn{4}{|c|}{ Percent } & \multirow{2}{*}{$\underset{\mathrm{Se}}{(\mathrm{ppm})}$} & \multicolumn{2}{|c|}{ Ratios } \\
\hline & & & eU & U & $\mathbf{C u}$ & $\mathrm{V}_{2} \mathrm{O}$ & & $\mathrm{eU} / \mathrm{U}$ & $\mathrm{V} / \mathrm{e} \mathrm{U}$ \\
\hline \multicolumn{10}{|c|}{ Uranium "ore" } \\
\hline $\begin{array}{l}251 \\
351 \\
350 \\
332\end{array}$ & $\begin{array}{r}b 4 \\
\mathbf{f 3 3} \\
\mathbf{f 3 2} \\
\mathbf{b 3}\end{array}$ & $\begin{array}{l}\text { Massive, fine- to medium-grained } \\
\text { pink, limy arkosic sandstone. May } \\
\text { contain green clay fragments, car- } \\
\text { bonized wood, chalcopyrite, and } \\
\text { uraniferous iron oxide(?). }\end{array}$ & $\begin{array}{l}0.11 \\
.044 \\
.038 \\
.022\end{array}$ & $\begin{array}{l}0.10 \\
.036 \\
.033 \\
.017\end{array}$ & $\begin{array}{r}0.01 \\
.02 \\
.02 \\
.02\end{array}$ & $\begin{array}{l}<0.1 \\
<.1 \\
<.1\end{array}$ & 3 & $\begin{array}{l}1.10 \\
1.22 \\
1.15 \\
1.30\end{array}$ & $\begin{array}{l}<1.27 \\
<2.54 \\
\end{array}$ \\
\hline
\end{tabular}

\begin{tabular}{|c|c|c|c|c|c|c|c|c|c|}
\hline \multicolumn{10}{|c|}{ Uranium-copper "ore" } \\
\hline $360^{1}$ & $\underset{b 4}{n e a r}$ & Coarse massive pink sandstone, chal- & 0.78 & 0.41 & $<0.46$ & $<0.2$ & 50 & 1.90 & $<0.26$ \\
\hline 352 & bI & 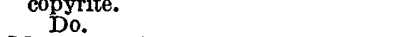 & .26 & .29 & 47 & .22 & & .76 & .47 \\
\hline$\cdot \frac{186}{353}$ & 111 & Micaceous siltstone and sandstone. & .18 & .20 & 1.27 & & & .90 & $-\cdots+$ \\
\hline 187 & I12 & Do. & .063 & .042 & .89 & & & $\begin{array}{l}0.00 \\
1.50\end{array}$ & $-\cdots$ \\
\hline $249^{-}$ & b10 & Brown arkosic sandstone. & .061 & .041 & 1.22 & & & 1.49 & $-\ldots$ \\
\hline 248 & b10 & Thin-bedded brown arkosic sandstone. & .058 & .049 & 1.37 & & & 1.14 & $-\ldots$ \\
\hline 247 & $\begin{aligned} \text { b10 } \\
\text { b7 }\end{aligned}$ & $\begin{array}{l}\text { Do. } \\
\text { Limy arkosic sandstone. }\end{array}$ & .051 & .036 & $\begin{array}{r}.79 \\
1.66\end{array}$ & 15 & 9 & $\begin{array}{l}1.42 \\
2.00\end{array}$ & \\
\hline $\begin{array}{r}204 \\
333 \\
-\end{array}$ & b4 & Limy arkosic sandstone with carbon. & .043 & .033 & $\begin{array}{r}1.00 \\
.62\end{array}$ & .12 & $\boldsymbol{y}$ & 1.30 & 1.56 \\
\hline
\end{tabular}

\begin{tabular}{|c|c|c|c|c|c|c|c|c|c|}
\hline \multicolumn{10}{|c|}{ Uranium-vanadium "ore" } \\
\hline 355 & $\mathbf{n 1}$ & Metatyuyamunite in micaceous sand- & 0.81 & 0.85 & -- & 2.92 & 50 & 0.95 & 2.0 \\
\hline $\begin{array}{l}34 y . \\
345 \\
346 \\
344 \\
343\end{array}$ & $\begin{array}{l}126 \\
f 23 \\
f 26 \\
\text { f2a } \\
\text { fis }\end{array}$ & $\begin{array}{l}\text { coarse mottled pink and black, limy, } \\
\text { arkosic sandstone with abundant } \\
\text { clay and rock fragments and carbon. } \\
\text { Some metatyuyamunite. }\end{array}$ & $\begin{array}{l}.20 \\
.075 \\
.046 \\
.042 \\
.015\end{array}$ & $\begin{array}{l}.18 \\
.038 \\
.041 \\
.027 \\
.006\end{array}$ & $\begin{array}{l}0.25 \\
.17 \\
.09 \\
.10 \\
.02\end{array}$ & $\begin{array}{r}2.07 \\
1.22 \\
1.36 \\
.90 \\
.29\end{array}$ & 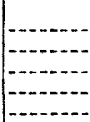 & $\begin{array}{r}1.10 \\
1.97 \\
1.10 \\
2.55 \\
2.50\end{array}$ & $\begin{array}{r}5.66 \\
8.97 \\
10.95 \\
11.95 \\
10.8\end{array}$ \\
\hline
\end{tabular}

${ }^{1}$ Colorimetric analyses by H. E. Crowe, R. R. Beins, and C. E. Thompson show approximately $150 \mathrm{ppm}$ As, $120 \mathrm{ppm} \mathrm{Mo,} \mathrm{and} 4 \mathrm{ppm} \mathrm{Ge}$.

The eU/U ratio (table 12) may give an indication of the amount of uranium that has been leached from the sample. The thorium content of these rocks is below $0.0 \mathrm{X}$, the spectrographic threshold, and can be ignored because the radioactivity of thorium is only about onefifth that of the same amount of uranium. Where uranium is in 
equilibrium with its daughter products, the ratio is 1.0. If selective leaching or precipitation of daughter products can be ignored, ratios much greater than 1.0 indicate recent leaching of uranium and ratios less than 1.0 indicate that uranium has been reprecipitated recently. This interpretation is less hazardous where the uranium content is appreciable (more than 0.05 percent), where the ratio is greater than 2.0 , and where there is corroborating field evidence.

The eU/U ratios (table 12) and the field evidence indicate that some leaching and redeposition of uranium occurs locally. The eU/U ratio changes from 2.0 to 4.0 in siltstone and shale, and apparently uranium is leached from the uraniferous shale and redeposited in adjacent sandstone in some areas.

\section{Geochemical PRospecting}

Geochemical prospecting techniques were used in the hope that deposits hidden under alluvium might be detected. A total of 457 botanical and 339 soil samples were collected. Wherever possible, soil and botanical samples were collected from the same place to determine which prospecting method was more satisfactory; the botanical samples were obtained from plant foliage to insure approximate uniformity of metal content and age of growth.

In general, the results were disappointing and neither geochemical nor botanical testing appears to be well suited to detecting small deposits of this type. A poor correlation between plant and soil data was obtained. This may be partly the result of the extraction of metals from the soil by plant roots. Several trenches dug beneath trees having a high copper or uranium content failed to disclose copper minerals or radioactivity in the bedrock. In area $I$, plant and soil data gave an entirely different pattern even though only the needles of ponderosa pine were sampled. The anomalies in content of copper in ponderosa pine outlined most of the copper deposits but neither the lead and zinc anomalies for ponderosa pine nor the anomalies for any of the three metals in soil samples were related to the ore bodies.

The reasons for the failure are: (1) prevalent surface wash, (2) different metal content of each rock type, and (3) the small. size of rock units and ore bodies which caused the root circle of plants to overlap several beds of different metal content. Samples of the foliage of ponderosa pine, pinyon, juniper, and scrub oak were collected. The metal content varied greatly but was higher in the pines than in the other trees.

The soil and ashed plant samples were analyzed for copper, lead, and zinc by rapid colorimetric methods. All three metals were determined in plant samples but only lead and zinc were determined in 255 soil samples. Uranium was determined by the fluorimetric method in 159 representative botanical samples. 


\section{ORIGIN}

In this section is postulated a tentative theory of origin of the mineral deposits that is consistent with the mineralogical, chemical, and field relationships. Such a theory is of necessity speculative; whether the ores were formed by the processes postulated remains to be demonstrated and depends on the correctness of assumptions that appear reasonable in the light of current physico-chemical knowledge but cannot be proved. The senior writer is primarily responsible for the ideas expressed in this section.

Briefly, the metals now concentrated in the mineral deposits are considered to have been derived from the Precambrian terrane and to have been concentrated by syngenetic sedimentary processes and, in the case of the uranium deposits in sandstone, reconcentrated by warm chemically active ground water with a possible juvenile component. Copper and uranium were partly separated because they were deposited in different chemical environments by different mechanisms. Copper was deposited as a sulfide by $\mathrm{H}_{2} \mathrm{~S}$, but uranium probably was adsorbed on a ferric hydroxide precipitate. The origin of each type of deposit is discussed separately in detail below and a summary is given at the end of the section.

\section{COPPER DEPOSITS}

The copper and associated uranium in the copper-bearing shale probably. were derived chiefly from Precambrian rocks. Many small copper deposits of pre-Pennsylvanian age are found in the Precambrian rocks of northern New Mexico. The metal probably was derived partly from these deposits but chiefly from acidic igneous rocks, which contain an average of 16 grams of copper per metric ton (0.0016 percent), according to Rankama and Sahama (1950, p. $697)$.

The carbonaceous copper deposits are local stagnant basin shale facies characterized by abundant carbonized plant remains and ferrous iron. The enclosing barren red rocks are colored by ferric oxides; they do not contain carbonaceous material, although plant imprints remain. The highest content of copper and uranium in the shale is found in shale with the most carbonaceous material. Copper probably was deposited in local swamps, lagoons, or lakes in an oxygendeficient environment which favored the carbonization of plant material and reduced iron to the ferrous state.

Copper, iron, and a little uranium from the Precambrian terrane probably were transported in solution by surface or ground waters to local anaerobic basins where copper and iron were precipitated as sulfides by hydrogen sulfide generated by the decay of organic material or the reduction of sulfates by anaerobic bacteria. A small part of the uranium was precipitated as uraninite. A little was 
probably adsorbed on carbon, ferric hydroxide, or humic compounds, but much of the uranium may have remained in solution or suspension to be deposited elsewhere.

The metal content and the form in which the metals were transported in solution are unknown but the metal content need not have been high if the process was long continued.

Microscopic study shows that carbonized wood was replaced first by pyrite and then by copper sulfides in the following order: chalcopyrite, bornite, chalcocite, blue chalcocite with excess $\mathrm{CuS}$, and covellite. Only pyrite and chalcocite are important quantitatively. The proportion of copper progressively increases and the proportion of iron and sulfur progressively decreases in each succeeding member of the series with the exception of sulfur in covellite. The amount of sulfide ion in solution seems to have been the critical factor determining the mineral content. The concentration of iron in solution was probably always greater than the concentration of copper because of its greater abundance in rocks and because it would be regenerated as iron-bearing sulfides were replaced by copper-bearing sulfides. Sulfide ion would be generated continuously by decay of organic matter or by reduction of sulfates by anaerobic bacteria, but the sulfide ion content of the solution fell as deposition of sulfides continued if the sulfur content of the minerals formed at each stage is a fair indication of the sulfide ion content of the solution. The solubility of iron sulfide is less than the solubility of copper sulfide, therefore pyrite began to precipitate first. Once the solubility product of copper sulfide was reached, however, mixed copper-iron sulfides began to precipitate and, as the sulfide ion concentration fell, sulfides progressively richer in copper were precipitated as a result of the greater affinity of copper for sulfide ion in acid aqueous solution. The mineralization process would be terminated by the exhaustion of sulfide or copper ion in solution or by changes in $\mathrm{pH}$ or oxidation potential. The very low uranium content (less than 0.01 percent) of the black copper-bearing shale beds can be explained partly by the low concentration and relatively high solubility of uranium in acid swamp water ( $\mathrm{pH} 4.0-4.5)$ and partly by precipitation of iron as a sulfide rather than as a flocculent hydroxide that could adsorb uranium. The near-absence of pitchblende, which might be expected to form in this environment, probably indicates that the solubility product was seldom exceeded because the oxidation potential was low enough. It must be tentatively concluded that the uranium and iron remained in solution or colloidal suspension in the excurrent waters to be deposited elsewhere. The writers are aware that many factors are ignored in the foregoing analysis.

The deposition of copper probably began with the establishment of anaerobic conditions, but it may have continued after the enclosing 
sediments were buried and during the period of diagenetic changes. Many chalcocite nodules have a concretionlike structure that indicates slow, gradual growth. Isolated masses of chalcocite occur in the cores of some limestone nodules. In strict terminology, these deposits are not syngenetic but rather semisyngenetic or penecontemporaneous with sedimentation and diagenetic processes. The presence of sulfide concretions, the association of copper with rocks deposited in an environment of putrefaction (Fäulnis) regardless of type, and the absence of copper in these same rock types deposited under other conditions strongly support a penecontemporaneous origin for the copper deposits. An alternate and, to the writers, less likely hypothesis would have the metals extracted from ground waters by adsorption or reduction after burial of the enclosing rocks. A third, and untenable, hypothesis would have the copper deposits formed by the accumulation of detrital grains of copper minerals derived from preexisting deposits. The association of copper with carbonaceous sedimentary rocks deposited in foul water is unmistakable whatever the origin of the deposits.

\section{URANIUM DEPOSITS}

Uranium and vanadium in addition to copper were probably derived from Precambrian rocks, but unlike the copper deposits the deposits of uranium and vanadium are, in part, epigenetic.

Precambrian pegmatite and (or) quartz veins contain uraniumbearing minerals in the Petaca, Ojo Caliente, Elk Mountain, Rociada, and Gallinas Canyon districts. The uranium-bearing minerals include uraninite, gummite, uranophane, samarskite, betafite, fergusonite, euxenite (?), and hatchettolite (Jahns, 1946, p. 61-68, 267, 277). These minerals contain niobium, tantalum, thorium, lithium, beryllium, or bismuth. The nearest of these districts, the Rociada district, is about 15 miles south of the Coyote district. Several radioactive anomalies have been detected in Precambrian and Pennsylvanian rocks by radioactivity surveys along the west side of the Coyote district. Part of the uranium came from the pegmatite but most of the uranium probably was derived from granitic rocks. Studies of the arkose derived from granite in the Powder River Basin of Wyoming show that possibly as much as two-thirds of the uranium in granitic rocks is present in disordered intergranular phases or is loosely held on the surface of mineral grains and can be released by mechanical disintegration without much chemical weathering (Harold Masursky, oral communication). Rankama and Sahama (1950, p. 634) report 3.963 grams of uranium per metric ton in granitic rocks and the wide distribution of radioactive anomalies in the granite and simple granite pegmatite of the Precambrian areas near Guadalupita suggests that this average figure may be too low for this granite. Investigators report the average vanadium content of igneous rocks to be 150 and 
315 grams of vanadium per metric ton (Rankama and Sahama, 1950, p. 596). The vanadium content of granite is much lower; investigators report between 1 and 30 grams per metric ton for granite or acidic igneous rocks (Rankama and Sahama, 1950, p. 596). The vanadium content is highest in gabbro and dolerite and part of the vanadium may have been derived from these rocks in the Precambrian terrane rather than for granite. Erosion of the source areas furnished enough uranium and vanadium to account for the amount concentrated in the deposits if an efficient process of concentration were available.

The uranium content of natural waters is very low except in the vicinity of uranium mines or districts where it may be from several hundred to several thousand parts per billion $\left(10^{\circ}\right)$. The uranium content of natural waters is very sensitive to changes in $\mathrm{pH}$. On the basis of present information, waters with a $\mathrm{pH}$ of 6.5 do not contain more than 1 to 6 parts per billion (Philip Fix, oral communication, 1954) but waters with a $\mathrm{pH}$ of 5.5 may contain several hundred parts per billion although the average in areas of uraniferous rocks probably is on the order of 10 . The uranium content of waters derived from the Precambrian terrane must have between the extreme limits of 1 and 100 parts per billion. A sample of domestic water from a well in the Guadalupita district contained 15 parts per billion and this figure does not seem unreasonable for natural waters from which the uraniferous shale beds were deposited. Considering the widespread occurrence of uraniferous rocks and sulfide deposits in the source areas, the waters in restricted drainages may have had a uranium content as high as 50 parts per billion and a $\mathrm{pH}$ as low as 4.0 for short periods of time where oxidizing sulfide deposits lowered the $\mathrm{pH}$. A large flow from an old tunnel draining the several square miles of a gold district has 80 parts per billion uranium and a $\mathrm{pH}$ of 2.5 (Philip Fix, oral communication, 1954). Using a figure of 10 parts per billion, the syngenetic uranium deposits in shale or siltstone represent a concentration ratio of 10,000 to 20,000 and the epigenetic deposits in sandstone represent a ratio higher than 100,000 . Such highconcentration ratios require efficient mechanisms of concentration operative in extremely dilute solutions. The adsorption mechanism postulated in this paper is believed adequate, although the adsorption of ferric hydroxide has not been fully tested. The scavenging power of activated charcoal and other compounds used in the laboratory to remove traces of deleterious ions from water shows the ability of some adsorptive compounds to remove metallic ions from extremely dilute solutions.

Uraniferous siltstone.-These deposits, though generally similar to the copper-bearing shale, differ from it in several ways. The uraniferous siltstone has a higher uranium ( 0.01 to 0.02 percent) and 
iron content and a much lower content of copper and carbonaceous matter than the black shale. The vanadium content is generally less than a few hundredths percent, only slightly higher than that of the copper-bearing shale. Copper is present as malachite, but sulfides or uranium minerals have not been found. Some uranium may be in detrital minerals containing rare earths, but most of it is probably adsorbed on iron oxides or on clay, mica, and carbonaceous material.

There is no reason to doubt a syngenetic or penecontemporaneous origin for the uraniferous siltstone. The uranium-bearing beds are persistent and the uranium content is relatively uniform. The differences in metal content between the uraniferous siltstone and the copper-bearing shale probably reflect differences in the conditions of deposition.

The hypothesis outlined in the following paragraph is suggested to explain the observed separation of uranium and copper and to indicate a direction for further research.

The uraniferous siltstone probably was deposited in a lagoon which had limited access to the sea. Anaerobic conditions were much less extreme than in the black shale and consequently the lagoonal water was more nearly normal. Both the $\mathrm{pH}$ and the oxidation potential were probably higher than in the stagnant-water environment in which the black shale was deposited. The absence of sulfide minerals, the lower copper content, and the higher uranium content of the siltstone probably are all indirect consequences of the low content of carbonaceous matter and hence the near absence of hydrogen sulfide. Under these conditions an effective mechanism for the precipitation of copper did not exist and the iron probably was precipitated as ferric hydroxide which readily adsorbs uranium rather than as a sulfide which does not. Uranium and copper minerals probably could not precipitate from the dilute near-neutral or slightly acid, mildly oxidizing waters and uranium was probably adsorbed on or coprecipitated with iron compounds such as ferric hydroxide that later dehydrated to form limonite. The precipitation of iron hydroxide and uranium was probably initiated by the mixing of excurrent water from the swampy flood plain and the stagnant-water basins in which the copper was deposited with the marine water of the lagoon. Colloids tend to be flocculated by sea water and recent data indicate that uranium is effectively removed from an acid, iron-rich solution ( $\mathrm{pH}$ 2.9) from which ferric hydroxide is precipitating when the solution is neutralized $(\mathrm{pH} \mathrm{6.0)}$. The acidity of swamp water $(\mathrm{pH}$ 4-4.5) would be neutralized by mixing with sea water (pH 8.1-9.6) and the uranium would be adsorbed on colloidal ferric hydroxide coagulated by sea water. If the sedimentation rate in the lagoon was low, a considerable concentration of uranium might occur over a long period of time. 
Uranium-vanadium deposits in sandstone.-The uranium-vanadium deposits in sandstone differ in several important respects from the copper deposits and the uraniferous siltstone, notably in the much higher uranium and vanadium contents. The metals, like those in the other deposits, are believed to have been derived from Precambrian rocks, but these deposits are epigenetic and probably formed long after the sandstone was deposited. A two-stage epigenetic origin is postulated.

Three processes by which the deposits could have formed are: (1) extraction and reconcentration from earlier low-grade syngenetic deposits by ground waters or hydrothermal solutions, (2) direct derivation from previous deposits or igneous rocks in the Precambrian source areas, or (3) addition of uranium and vanadium from a magnetic source. None of these possibilities can be disproved but the first is most probable and the last is unlikely.

The absence of uranium, vanadium, and copper along the major faults and the lack of a known magmatic source for these metals argue against a simple hydrothermal origin; however, hydrothermal solutions probably were active in the district as shown by the sericitization of sheared arkose and the replacement of detrital quartz and feldspar by calcite and hematite. No indication of the source of the hydrothermal solutions has been found; they may have been derived from the basalt plugs east of the district or from an unknown body at depth. A relationship cannot be demonstrated between sericitized ground and uranium deposits and the role of a hydrothermal component in the formation of the deposits can be questioned if the less clear-cut alteration changes described in the following paragraphs can be explained in another way.

Normal surface or ground water probably could not cause the replacement of quartz and feldspar by calcite. If calcite were introduced by a normal cementation process the sand grains should be nearly in contact, but the grains are corroded and isolated where replacement is advanced. The high manganese content of some calcite and the extensive replacement of quartz and feldspar in most uranium deposits seem to require the introduction of some calcite by chemically active fluids, perhaps at a higher temperature than normal for ground water. If this conclusion is correct it is reasonable to ascribe the calcitization to an early effect of the solution responsible for the deposition of iron and uranium.

A direct relationship between the degree of hematitic alteration and the uranium is shown by the field and laboratory evidence. Hematite, nontronite, and the uraniferous ferric oxide are considered products of warm, chemically active ground water with a possible juvenile component. The presence of fresh feldspar and chalcopyrite on the outcrops suggests that the hematitic alteration is not related 
to surface oxidation. The iron in nontronite, a montmorillonite-type clay with ferric iron, cannot be added without destroying the structure of the clay mineral, for example, by the adsorption of iron on a montmorillonite clay in normal ground water. The iron must have been introduced at the time nontronite was formed-and the formation of nontronite, except by the weathering of ferromagnesian minerals, probably requires a high ferric iron concentration and temperatures higher than those of normal ground waters.

To explain the field and laboratory evidence the writers tentatively postulate a two-stage epigenetic theory of origin: that the uranium deposits in sandstone are epigenetic and were leached and redeposited from earlier syngenetic uranium deposits containing from 0.005 to 0.03 percent uranium. The evidence is not conclusive and the hypothesis is necessarily speculative.

Barren chemically active hydrothermal(?) solutions are believed to have migrated along the Laramide(?) faults and through permeable beds. The warm, active acid solutions sericitized the arkoses near the channels and leached iron, uranium, vanadium, and calcium from the low-grade uraniferous rocks through which they moved. Much of the uranium in the shale probably was loosely held by adsorption and hence was readily removed from the shale adjacent to the faults. The ability of such hydrothermal solutions to sericitize the rocks and to leach iron and uranium is shown by Phair's study (1952) of the altered radioactive quartz bostonite in the Front Range of Colorado. Phair found that an appreciable part of the uranium and iron were leached from the altered quartz bostonite dikes near the pitchblende deposits.

The acid solutions spread out in permeable sandstone beds and merged with the ground-water system whose circulation may have been reactivated by folding. The ground water with its juvenile(?) component flushed the sandstone and picked up more metal. The movement of the ground water was controlled by the permeability, porosity, and structure of the aquifers. In favorable sedimentary structures, uranium-vanadium and iron were deposited by change in $\mathrm{pH}$, oxidation potential, complex-ion destruction, or flocculation of a colloidal suspension by dilution or mixing of solutions of different characteristics. It is suggested that uranium compounds could not form under the existing conditions and the uranium and rare earths were adsorbed on or coprecipitated with ferric hydroxide formed when the originally acid solution was neutralized by dilution or by reaction with calcite. The ferric hydroxide might have remained in suspension and continued to move with the ground waters until flocculated by some mechanism, such as mixing with waters containing dissolved salts or strained out by decrease in pore size of the sandstone. The flocculent precipitate may have continued to adsorb uranium from mi- 
grating ground waters until the unbalanced charges were fully neutralized. Precipitation of ferric hydroxide begins at a $\mathrm{pH}$ of 3.0-3.5 and virtually all the uranium will be removed from an ironrich solution by the time a $\mathrm{pH}$ of 6 is reached. The marked concentration of rare earth in uraniferous iron oxides (samples 251 and 89, table 11) might also be explained by adsorption or coprecipitation on ferric hydroxide because their behavior is generally similar to the behavior of uranium. Vanadium, where present, seems to be in the clay minerals or adsorbed on carbonized wood. Probably most of the vanadium is in a vanadium hydromica formed by incorporation of vanadium in the structure during the recrystallization of an existing clay mineral. The proportions of uranium, vanadium, and copper were controlled by the characteristics of the sediments and the density, temperature, $\mathrm{pH}$, oxidation potential, and metal content of the solution.

The principal evidence against a syngenetic origin for the uraniumvanadium deposits in sandstone is the difficulty in explaining the sericitization of sheared arkose, the extensive replacement of quartz and feldspar by calcite and hematite, and the presence of both early sulfides containing ferrous iron and late ferric iron oxides containing uranium and rare earths. The uranium content of the uraniferous siltstone probably represents the maximum that can be expected in syngenetic deposits formed under conditions that existed in the Coyote district, unless the uranium content of the water was abnormally high at certain times. An abnormally high uranium content in water can be expected only where the water is acid and waters more acid than those that deposited the black shale ( $\mathrm{pH} 4.0-4.5)$ could hardly be expected in a terrane containing limestone. The uranium content of the black shale and uraniferous siltstone is low as comared to that of the sandstone deposits but the total amount is much greater. The uranium content of a syngenetic uranium deposit is limited by the sedimentation rate and by the suggestion that uranium is readily removed by absorption in slightly acid or neutral waters and hence is dispersed. The uranium content of the syngenetic deposits in the Coyote district is comparable to that of most other syngenetic deposits except for a few deposits like the presumably syngenetic Swedish kolm (ash-rich coal(?)) that have a much higher organic content.

Given a sufficient volume of solution and an efficient mechanism of concentration, uranium deposits might be formed from very dilute solutions, even from the ground waters existing in the district today. A water sample from a well in the central part of the district contained 0.015 parts per million uranium (Zeller and Baltz, 1954, p. 11). Several high uranium concentrations in ponderosa pine in area $E$ were not paralleled by anomalies in the bedrock and the uranium probably 
was extracted from dilute ground water percolating through the sheared ground.

Although a few clastic grains containing chalcopyrite and a black substance have been seen in the uranium ore, microscopic examination reveals that virtually all the uranium is later than the calcite cement. The absence of niobium, tantalum, thorium, and other elements derived from the uranium-bearing pegmatite and the scarcity of the resistant minerals of these elements proves that the amount of uranium, vanadium, and rare earths contributed by the heavy minerals is negligible and indicates that most of the uranium was not-derived from the pegmatite.

\section{SUMMARY}

Copper and smaller amounts of uranium and vanadium freed by erosion of the Precambrian terrane were transported to the basins of depositions in solution. The metals were removed from solution by different mechanisms operating under different conditions and this caused a partial separation of metals. The copper and part of the iron in solution were precipitated as sulfide in local anaerobic basins where carbonaceous shale was accumulating but most of the uranium and some iron remained in the still-acid excurrent water, either in solution or suspension until the $\mathrm{pH}$ approached 6 by dilution in lakes or lagoons or by reaction with limy sediments. A large part of the uranium probably was dispersed upon neutralization by absorption in clays but a considerable concentration of uranium occurred where the still-acid excurrent waters mingled with sea water in a marine lagoon where the sedimentation rate was slow. Here colloidal suspensions of ferric hydroxide would be coagulated and the remaining iron would be precipitated as ferric hydroxide. The uranium forced out of solution by increase in $\mathrm{pH}$ upon dilution would be absorbed on or coprecipitated with the iron hydroxide, which later dehydrated to form hematite or limonite.

Following Laramide(?) orogeny warm acid ground waters with a chemically active (hydrothermal?) component migrating through sheared ground and along faults leached lime, iron, uranium, vanadium, and rare earths from the uraniferous sedimentary rocks and mingled with the ground waters in the sandstone aquifers. The waters were neutralized by dilution by reaction with the calcite cement of the sandstone, and uranium and rare earths were adsorbed on ferric hydroxide precipitated in favorable sedimentary structures.

\section{COMPARISON WITH OTHFR COPPER-URANIUM DEPOSITS}

Copper-uranium deposits similar to the deposits in the Coyote district have been found in arkosic red beds, at least partly equivalent to the Sangre de Cristo formation, in several areas marginal to the ancestral Rocky Mountains. 
The Cutler formation contains small copper-uranium deposits in the Big Indian Wash district and the Lockhart Canyon-Indian Creek area of Utah (G. P. Dix, written communication, 1953). The Abo formation, equivalent in part to both the Cutler formation and the Sangre de Cristo formation, contains copper deposits in the Scholle, Zuni Mountain, and Jemez Springs districts of New Mexico. Uranium has been reported from the Scholle and Jemez Springs district. Small copper-uranium deposits have been found in the Maroon formation, a partial equivalent of the Sangre de Cristo formation in the Huerfano Park añd La Veta Pass areas, Huerfano and Costilla Counties, Colo. The Garo property, Park County, Colo. (Gott, written communication, 1951), has produced a small tonnage of uranium ore from sandstone in the Maroon (?) formation. The Cutler, Abo, Sangre de Cristo and Maroon formations form a continuous arkosic red bed unit. The deposits in the Lockhart Canyon-Indian Creek area of Utah and the deposits in the Maroon formation of Colorado resemble the sandstone deposits in the Coyote district. The deposits near Garo and La Veta, Colo., and in the Coyote district have a similar structural setting along the flanks of the Sangre de Cristo Mountain system.

The occurrence of similar deposits in arkose deposits on the flanks of areas of Pennsylvanian uplift in widely separated areas suggests that other similar deposits exist. The similarities to the deposits of the Coyote district further suggest that ore guides at Guadalupita may be applicable to prospecting in other areas.

Copper deposits of the red-bed type, some of which contain small amounts of uranium, are present in the Poleo or Aqua Zarca sandstone lentils of the Chinle formation of Triassic age in the Cuba, Abiquiu, Cobre Basin, Copper City, and Gallinas districts, all of which surround the Nacimiento uplift in New Mexico. Uranium has been reported from the Chinle formation near Coyote north of the Nacimiento uplift and near Sabinosa along the Canadian River east of Las Vegas, N. Mex. These deposits are similar to those described in this report. Uranium deposits might be found in sandstone which does not contain appreciable quantities of copper in other red-bed copper districts.

\section{GUIDES TO ORE DEPOSITS}

The ore guides described below pertain to the Coyote district but they may have a wider application as indicated in the preceding section.

In prospecting for copper deposits special attention should be directed to parts of the Sangre de Cristo formation that contain abundant limestone or gray shale beds, because the ore-bearing black shale lenses are enclosed in gray shale and may grade into limestone. 
The uranium deposits are in one or more brown or gray fluvial sandstone beds near the middle of the Sangre de Cristo formation. The outcrops are inconspicuous and a radiation counter is necessary for prospecting. Chalcopyrite, malachite, or black vanadium minerals and the distinctive pink color of the sandstone are the best ore guides but are easily overlooked. The most favorable places in sandstone are cut-and-fill structures, especially where the sandstone is coarse and contains abundant gray or black clay and foreign rock fragments, and carbonized wood. The red (or maroon) sediments probably can be ignored because evidence of mineralization has not been found in them.

\section{CONCLUSIONS}

The uranium content of the copper deposits and the uraniferous siltstone is too low to permit the recovery of uranium under presentday conditions. The uranium deposits in sandstone are small, but some production might be expected under favorable conditions. Further exploration probably will result in an increase of uranium reserves but no large deposits are expected. Geochemical prospecting methods are not suitable because of the steep dip of the beds and small size of the mineral deposits.

A large part of the value of the present investigation, in the opinion of the writers, lies in the genetic implications and practical application of the relationships discovered between copper deposits and the uranium-vanadium deposits. Some of the more important relationships, chosen for their possible application to the search for uranium deposits in other areas, are:

(1) The largest copper deposits are in local black carbonaceous shale lenses and contain a negligible amount of uranium.

(2) The only commercial-grade uranium deposits are low in copper. They occur in several gray or brown fluvial sandstone beds and are localized by small sedimentary structures similar to those in the Morrison and Shinarump formations. It is suggested that uranium deposits may be found in fluvial sandstone in other "redbed" copper districts but that the conditions favorable for the formation of commercial-grade uranium deposits are not the same as those favorable for red-bed copper deposits.

(3) The copper-bearing shale and the uraniferous siltstone are essentially syngenetic but the uranium-vanadium deposits in sandstone probably are much younger and may have formed from low-grade syngenetic deposits by ground water having a hydrothermal(?) component following Laramide(?) orogeny. If this theory of origin is correct, the search for uranium deposits in arkosic red-bed units should be directed to areas where favorable sandstone beds are folded 
or faulted, particularly where they surround younger intrusives. It is suggested that undiscovered uranium deposits may exist along the flanks of the present mountains where limy sandstone beds in sedimentary rocks of the Cutler, Abo, Sangre de Cristo, and Maroon formations or formations of similar lithology are exposed.

(4) It is suggested that the coprecipitation with or adsorption on ferric hydroxide offers a mechanism by which uranium deposits can form even where uranium compounds (minerals) cannot form. This process is operative in acid oxidizing solutions between a $\mathrm{pH}$ of 3 and 6 and applies equally to syngenetic and epigenetic deposits. It is suggested that the ferric hydroxide is usually precipitated by reaction with calcite. The same adsorption process is believed to cause the concentration of the rare earths. The uranium in both the siltstone and the sandstone probably was concentrated by this mechanism. If the foregoing is true, the search for large low-grade uranium deposits need not be limited to sedimentary rocks rich in organic material but should be extended to certain fine-grained sedimentary rocks containing iron oxides formed in the manner described.

(5) The distinct pink color imparted by hematite introduced with uranium is the best ore guide to the sandstone deposits. The difference between it and the red, chocolate, or maroon color of the barren red beds is slight but distinctive.

\section{LITERATURE CITED}

Bachman, G. O., 1953, Geology of a part of northwestern Mora County, N. Mex. : U. S. Geol. Survey, Oil and Gas Inv. Map,.OM 137.

Brill, K. G., Jr., 1952, Stratigraphy in the Permo-Pennsylvanian Zeugogeosyncline of Colorado and Northern New Mexico: Geol. Soc. America Bull., v. 63, p. 809-880.

Edwards, A. B., 1947, Textures of the ore minerals and their significance: Australasian Inst. Min. Metallurgy, Melbourne, $185 \mathrm{p}$.

Gott, G. B., and Erickson, R. L., 1952, Reconnaissance of uranium and copper deposits in parts of New Mexico, Colorado, Utah, Idaho and Wyoming: U. S. Geol. Survey Circ. 219.

Harley, G. T., 1940, The geology and ore deposits of northeastern New Mexico: New Mexico Bur. Mines Bull. 15, 104 p.

Jahns, R. H., 1946, Mica deposits of the Petaca district, Rio Arriba County, N. Mex. : New Mexico Bur. Mines Bull. 25, 294 p.

Lasky, S. G., and Wooton, T. P., 1933, The metal resources of New Mexico and their economic features : New Mexico Bur. Mines Bull. 7, 178 p.

Lindgren, Waldemar, Graton, L. C., and Gordon, C. H., 1910, Ore deposits of New Mexico: U. S. Geol. Survey Prof. Paper 68, 361 p.

Northrup, S. A., Sullwold, H. H., Jr., MacAlpin, A. J., and Rogers, C. P., Jr., 1946, Geologic maps of a part of the Las Vegas basin and of the foothills of the Sangre de Cristo Mountains, San Miguel and Mora Counties, N. Mex, : U. S. Geol. Survey, Oil and Gas Inv. Prelim. Map 54. 
COPPER AND URANIUM DEPOSITS, COYOTE DISTRICT, N. MEX. 395

Phair, George, 1952, Radioactive Tertiary porphyries in the Central City district, Colo., and their bearing on pitchblende deposition: U. S. Geol. Survey Trace Elements Inv. Rept. 247, U. S. Atomic Energy Tech. Inf. Service, Oak Ridge, Tenn.

Rankama, Kalervo, and Sahama, T. G., 1950, Geochemistry: Chicago, Univ. Chicago Press, 911 p.

Read, C. B., and Wood, G. H., 1947, Distribution and correlation of Pennsylvanian rocks in late Paleozoic sedimentary basins of northern New Mexico: Jour. Geol., v. 55, p. 220-237.

Zeller, H. D., and Baltz, E. H., Jr., 1954, Uranium-bearing copper deposits in the Coyote district, Mora County, N. Mex. : U. S. Geol. Survey Circ. 334. 
$=\cdots$ 


\section{INDEX}

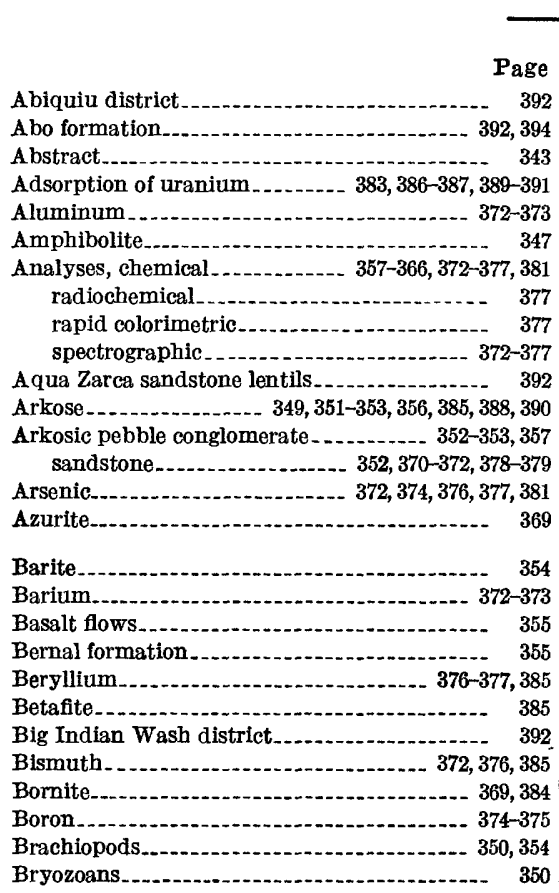

Calcite. $354,369,380,388,389,390,391$ Calcium

Carbonaceous shale._._. _. _._. $356,357,367-368$ Carbonized wood.......... 369-370, 383-384, 393

Chacon............ 349

Chalcocite................... 354, 369,377,384, 385

Chalcopyrite........... 371, 379, 380, 384, 388, 391, 393

Chinle formation......... 392

Chromium . 374-375

Cobalt_._._. 374-375

Cobre Basin district.

Copper-_._._. 356-370, 379, 380-385, 387, 388, 390-393

Copper City district....

Corals. .

Covellite........ 369, 377, 384

Coyote Creek.

Cuba district._. 392

Cutler formation.

Cyelothems............... 354

Diabase. . .

Digenite-_._._. 369

Diorite........... 347

Elk Mountain district_._.

Equivalent uranium (eU) .......... . . . 357-368, $371,372-373,381-382$

Euxenite

Feldspar _. . .

Fergusonite.............. 385

Fluviatile sandstone unit_...

Gallinas district ......................... 392

Gallium _..._. $374-375$

Garo uranium property._._._._. 392

Geochemical prospecting anomalies._._._._. $\quad 382$

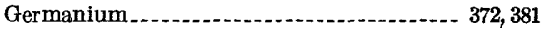

Glorieta sandstone member............ 347,355

Gneiss..................... 347

Granite._._._........ 347

Gummite._._._. 385

Hatchettolite........... 385

Hematite................ 379, 380, 388, 390, 391, 394

Huerfano Park ................... 392

Hydrothermal leaching. . . . . . 389

Hypselentoma........ 354

Ionium_.............. 371,376

Iron _........ _... 371, 372-373, 383, 388, 389, 391

Jarosa syncline

Jemez Springs district._..... 392

Kolm._. 390

La Veta Pass area_......... 392

Leaching of uranium ............. 371

Lead .

Limestone............... 349, 354, 357, 366, 392

Limonite _...... 391

Lithium...

Lockhart Canyon-Indian Creek area, Utah...- 392

Magdalena group................. 346, 349-350, 356

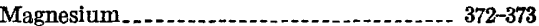

Malachite _. . $369,371,379,387,393$

Manganese..._._._._._._._._._. 374-375, 388

Marble Falls limestone.................... 350

Maroon formation............ 392.394

Metatyuyamunite_._._._._. 370, 371, 378-379

Micaceous sandstone.............. 357

Mollusks.

Molybdenum _........... 374-375, 381

Montmorillonite.... 389

Morrison formation........ 381, 393

Muscovite...... 380

Nacimiento uplift _. .

Nickel....

Niobium _. _ _.

Nontronite _......

Ocate anticline

area................ 345

Ojo Caliente district__._. 385

Paragenetic sequence._. 385 


\begin{tabular}{|c|c|}
\hline Page & e \\
\hline $347,385,391$ & Shinarump formation \\
\hline (n) & Silicon......... 372-373 \\
\hline (n) & Siltstone \\
\hline itchblende....... & Silver \\
\hline otassium & Smithwick shale. \\
\hline oleo sandstone lentils. $\ldots \ldots \ldots \ldots \ldots \ldots \ldots \ldots$ & Sodium \\
\hline 369,384 & Strontium........... \\
\hline $380,385,388,390$ & 385,391 \\
\hline e... & $\begin{array}{l}\text { Thorium } \\
\text { Titanium }\end{array}$ \\
\hline adioactive equilibrium............... 371, 381-382 & Tourmal \\
\hline hs & 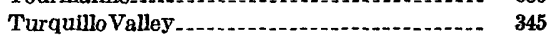 \\
\hline ountains. & aity \\
\hline 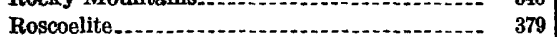 & Uraninite..... $369,379,385$ \\
\hline 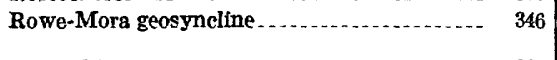 & $\begin{array}{r}\text { Uranium } \\
\qquad 370,371,378-380,382,383,385-386,388-394\end{array}$ \\
\hline (- & Uranium daughter elements.......... \\
\hline Andres formation & Uranophane \\
\hline andia formation....... 349 & \\
\hline andstone & $370,376-377,378,379,381,3$ \\
\hline $\begin{array}{l}\text { angre de Cristo formation } \\
\qquad 347,349-355,356,378,391-394\end{array}$ & $\begin{array}{l}\text { Vanadium oxide } \\
\text { Vitrain }\end{array}$ \\
\hline sandstone...................... 355 & Volborthite........ 369 \\
\hline candium & Volcanic plugs....... 355 \\
\hline hist. & Water, uranium content..... \\
\hline trict & \\
\hline $78,379,386,387,391,392$ & Zirconium \\
\hline ale, copper-bearing carbonaceous........... & 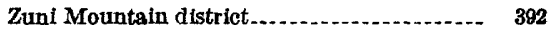 \\
\hline
\end{tabular}

